\title{
Asymmetric Synthesis of All-Carbon Benzylic \\ Quaternary Stereocenters via Copper-Catalyzed \\ Conjugate Addition of Dialkylzinc Reagents to \\ 5-(1-Arylalkylidene) Meldrum's Acids
}

Eric Fillion* and Ashraf Wilsily

efillion@uwaterloo.ca

Supporting Information 2

Substrate ${ }^{1} \mathrm{H}$ and ${ }^{13} \mathrm{C}$ NMR Spectra 


\section{Table of Contents}

${ }^{1} \mathrm{H}$ NMR spectrum for 2,2-dimethyl-5-(1-phenylethylidene)-1,3-dioxane-4,6-dione (1a) 4

${ }^{13} \mathrm{C}$ NMR spectrum for 2,2-dimethyl-5-(1-phenylethylidene)-1,3-dioxane-4,6-dione (1a) 5

${ }^{1} \mathrm{H}$ NMR spectrum for 2,2-dimethyl-5-[1-(2-naphthyl)ethylidene]-1,3-dioxane-4,6-dione (1b) 6

${ }^{13}$ C NMR spectrum for 2,2-dimethyl-5-[1-(2-naphthyl)ethylidene]-1,3-dioxane-4,6-dione (1b)

${ }^{1} \mathrm{H}$ NMR spectrum for 5-[1-(2-furyl)ethylidene]-2,2-dimethyl-1,3-dioxane-4,6-dione (1c) 8

${ }^{13} \mathrm{C}$ NMR spectrum for 5-[1-(2-furyl)ethylidene]-2,2-dimethyl-1,3-dioxane-4,6-dione (1c) 9

${ }^{1} \mathrm{H}$ NMR spectrum for 2,2-dimethyl-5-[1-(4-methylphenyl)ethylidene]-1,3-dioxane-4,6-dione (1d) 10

${ }^{13} \mathrm{C}$ NMR spectrum for 2,2-dimethyl-5-[1-(4-methylphenyl)ethylidene]-1,3-dioxane-4,6-dione (1d) 11

${ }^{1} \mathrm{H}$ NMR spectrum for 2,2-dimethyl-5-[1-(4-phenylphenyl)ethylidene]-1,3-dioxane-4,6-dione (1e) 12

${ }^{13} \mathrm{C}$ NMR spectrum for 2,2-dimethyl-5-[1-(4-phenylphenyl)ethylidene]-1,3-dioxane-4,6-dione (1e) 13

${ }^{1} \mathrm{H}$ NMR spectrum for 5-[1-(4-chlorophenyl)ethylidene]-2,2-dimethyl-1,3-dioxane-4,6-dione (1f) 14

${ }^{13} \mathrm{C}$ NMR spectrum for 5-[1-(4-chlorophenyl)ethylidene]-2,2-dimethyl-1,3-dioxane-4,6-dione (1f) 15

${ }^{1} \mathrm{H}$ NMR spectrum for 5-[1-(4-bromophenyl)ethylidene]-2,2-dimethyl-1,3-dioxane-4,6-dione (1g) 16

${ }^{13} \mathrm{C}$ NMR spectrum for 5-[1-(4-bromophenyl)ethylidene]-2,2-dimethyl-1,3-dioxane-4,6-dione (1g) 17

${ }^{1} \mathrm{H}$ NMR spectrum for 5-[1-(4-fluorophenyl)ethylidene]-2,2-dimethyl-1,3-dioxane-4,6-dione (1h) 18

${ }^{13}$ C NMR spectrum for 5-[1-(4-fluorophenyl)ethylidene]-2,2-dimethyl-1,3-dioxane-4,6-dione (1h) 19

${ }^{1} \mathrm{H}$ NMR spectrum for 2,2-dimethyl-5-1-[4-(trifluoromethyl)phenyl]ethylidene-1,3-dioxane-4,6-dione (1i) 20

${ }^{13}$ C NMR spectrum for 2,2-dimethyl-5-1-[4-(trifluoromethyl)phenyl]ethylidene-1,3-dioxane-4,6-dione (1i) 21

${ }^{1} \mathrm{H}$ NMR spectrum for 5-1-[4-(benzyloxy)phenyl)] ethylidene-2,2-dimethyl-1,3-dioxane-4,6-dione (1j) 22

${ }^{13} \mathrm{C}$ NMR spectrum for 5-1-[4-(benzyloxy)phenyl)]ethylidene-2,2-dimethyl-1,3-dioxane-4,6-dione (1j) 23

${ }^{1} \mathrm{H}$ NMR spectrum for 2,2-dimethyl-5-[1-(3-methylphenyl)ethylidene]-1,3-dioxane-4,6-dione (1k) 24

${ }^{13}$ C NMR spectrum for 2,2-dimethyl-5-[1-(3-methylphenyl)ethylidene]-1,3-dioxane-4,6-dione (1k) 25

${ }^{1} \mathrm{H}$ NMR spectrum for 5-[1-(3-chlorophenyl)ethylidene]-2,2-dimethyl-1,3-dioxane-4,6-dione (11) 26

${ }^{13} \mathrm{C}$ NMR spectrum for 5-[1-(3-chlorophenyl)ethylidene]-2,2-dimethyl-1,3-dioxane-4,6-dione (11) 27

${ }^{1} \mathrm{H}$ NMR spectrum for 5-1-[3-(benzyloxy)phenyl)] ethylidene-2,2-dimethyl-1,3-dioxane-4,6-dione (1m) 28

${ }^{13}$ C NMR spectrum for 5-1-[3-(benzyloxy)phenyl)] ethylidene-2,2-dimethyl-1,3-dioxane-4,6-dione (1m) 29

${ }^{1} \mathrm{H}$ NMR spectrum for 5-[1-(3,4-dichlorophenyl)ethylidene]-2,2-dimethyl-1,3-dioxane-4,6-dione (1n) 30

${ }^{13}$ C NMR spectrum for 5-[1-(3,4-dichlorophenyl)ethylidene]-2,2-dimethyl-1,3-dioxane-4,6-dione (1n) 31

${ }^{1}$ H NMR spectrum for 2,2-dimethyl-5-[1-(2-methylphenyl)ethylidene]-1,3-dioxane-4,6-dione (10) 32

${ }^{13} \mathrm{C}$ NMR spectrum for 2,2-dimethyl-5-[1-(2-methylphenyl)ethylidene]-1,3-dioxane-4,6-dione (1o) 33

${ }^{1} \mathrm{H}$ NMR spectrum for 5-[1-(2-chlorophenyl)ethylidene]-2,2-dimethyl-1,3-dioxane-4,6-dione (1p) 34

${ }^{13} \mathrm{C}$ NMR spectrum for 5-[1-(2-chlorophenyl)ethylidene]-2,2-dimethyl-1,3-dioxane-4,6-dione (1p) 35

${ }^{1} \mathrm{H}$ NMR spectrum for 5-1-[2-(benzyloxy)phenyl)] ethylidene-2,2-dimethyl-1,3-dioxane-4,6-dione (1q) 36

${ }^{13} \mathrm{C}$ NMR spectrum for 5-1-[2-(benzyloxy)phenyl)]ethylidene-2,2-dimethyl-1,3-dioxane-4,6-dione (1q) 37

${ }^{1} \mathrm{H}$ NMR spectrum for 5-[1-(4-chlorophenyl)pentylidene]-2,2-dimethyl-1,3-dioxane-4,6-dione (1r) 38

${ }^{13} \mathrm{C}$ NMR spectrum for 5-[1-(4-chlorophenyl)pentylidene]-2,2-dimethyl-1,3-dioxane-4,6-dione (1r) 39 
${ }^{1} \mathrm{H}$ NMR spectrum for 2,2-dimethyl-5-(2-methyl-1-phenylpropylidene)-1,3-dioxane-4,6-dione (1s)

${ }^{13} \mathrm{C}$ NMR spectrum for 2,2-dimethyl-5-(2-methyl-1-phenylpropylidene)-1,3-dioxane-4,6-dione (1s)

${ }^{1} \mathrm{H}$ NMR spectrum for 5-[1-(4-chlorophenyl)propylidene]-2,2-dimethyl-1,3-dioxane-4,6-dione (1t)

${ }^{13} \mathrm{C}$ NMR spectrum for 5-[1-(4-chlorophenyl)propylidene]-2,2-dimethyl-1,3-dioxane-4,6-dione (1t)

${ }^{1} \mathrm{H}$ NMR spectrum for 5-(2,3-dihydro- $1 H$-1-indenyliden)-2,2-dimethyl-1,3-dioxane-4,6-dione (4a) 44

${ }^{13} \mathrm{C}$ NMR spectrum for 5-(2,3-dihydro-1H-1-indenyliden)-2,2-dimethyl-1,3-dioxane-4,6-dione (4a)

${ }^{1} \mathrm{H}$ NMR spectrum for 5-(5-chloro-2,3-dihydro-1H-1-indenyliden)-2,2-dimethyl-1,3-dioxane-4,6-dione (4b) 46

${ }^{13} \mathrm{C}$ NMR spectrum for 5-(5-chloro-2,3-dihydro- $1 \mathrm{H}$-1-indenyliden)-2,2-dimethyl-1,3-dioxane-4,6-dione (4b) 


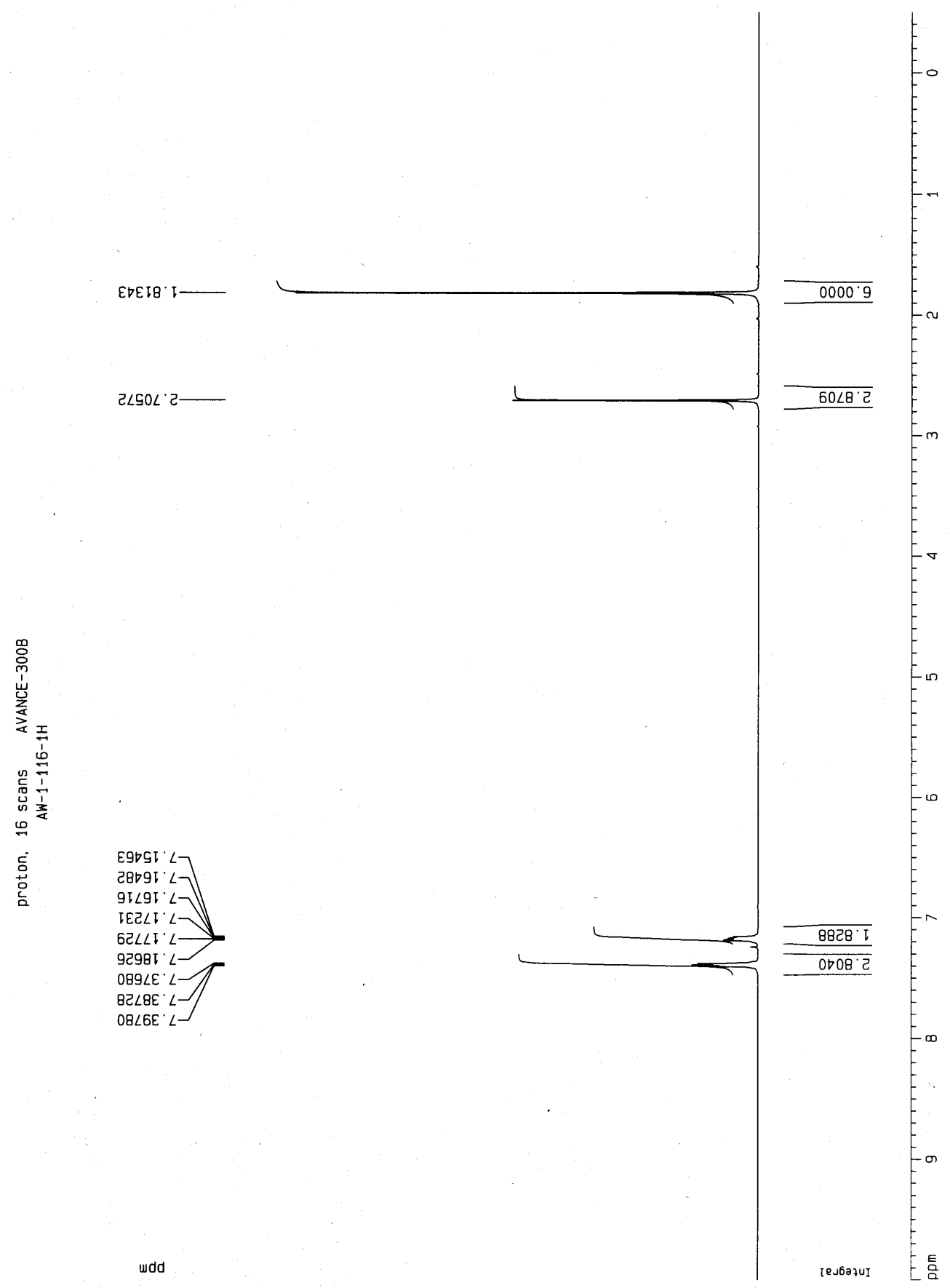




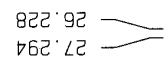

$8 \angle 9^{\circ} 9 \angle$

$100 . \angle L$

$92 \downarrow \circ L>$

莶

OL'EOI-

928.915

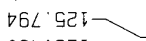

OEV. $8 \mathrm{CL} \longrightarrow$

1998.851

$\nabla 89^{\circ}$ IVI

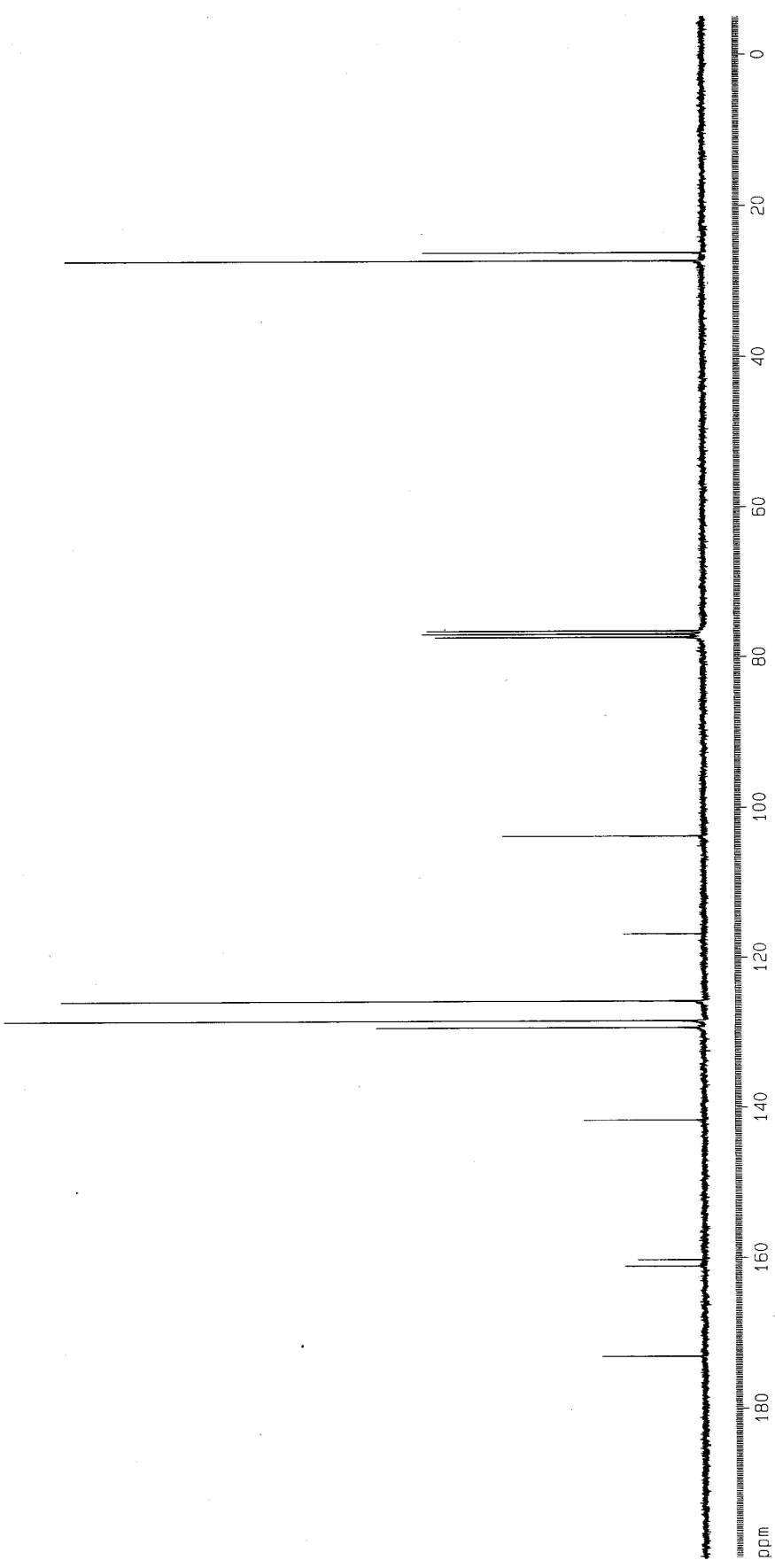

wdd

IDC.095

9นT.

$890^{\circ} E \angle I \longrightarrow$ 


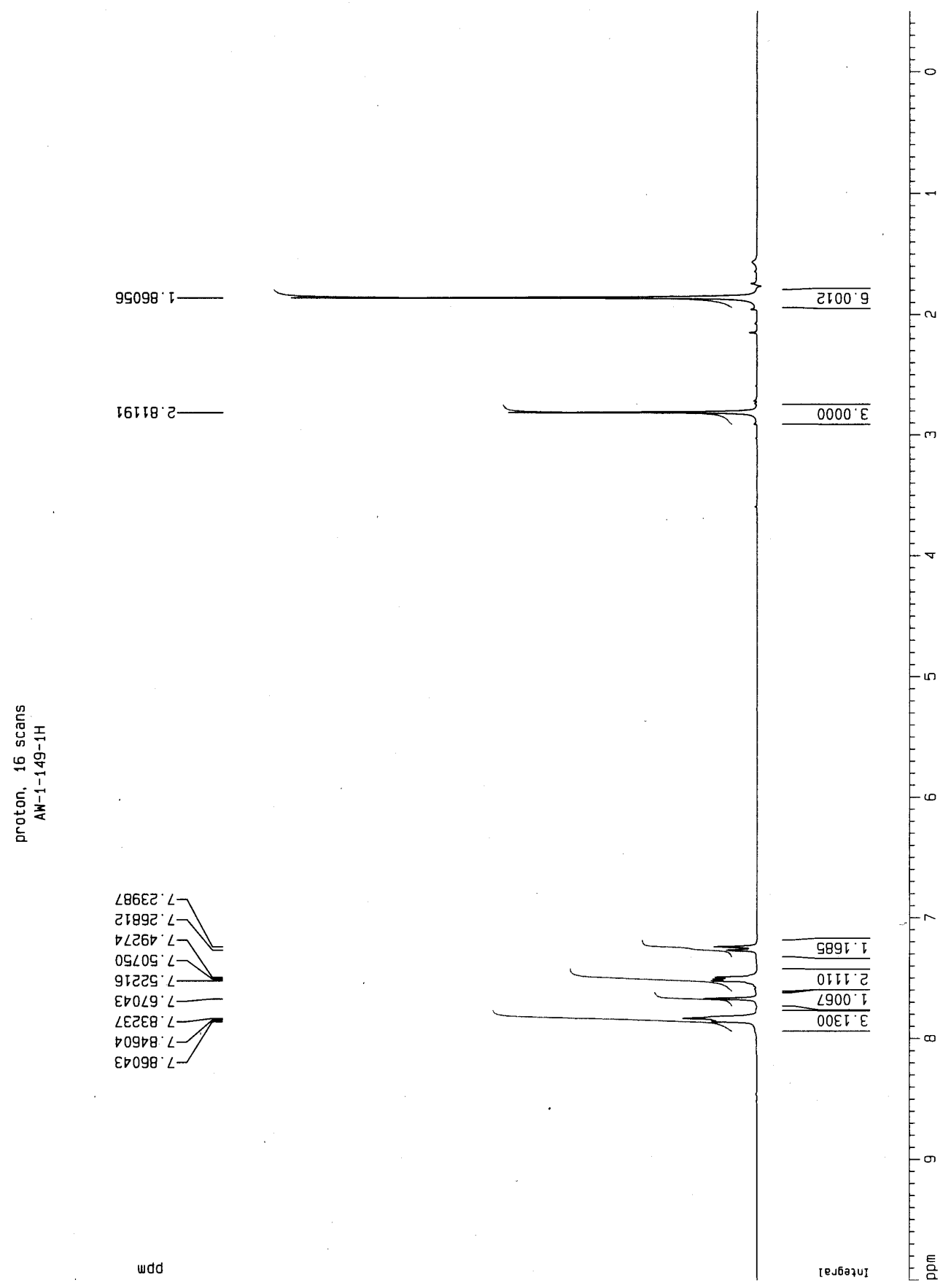


${ }_{\nabla 98}^{980} \cdot 92=$

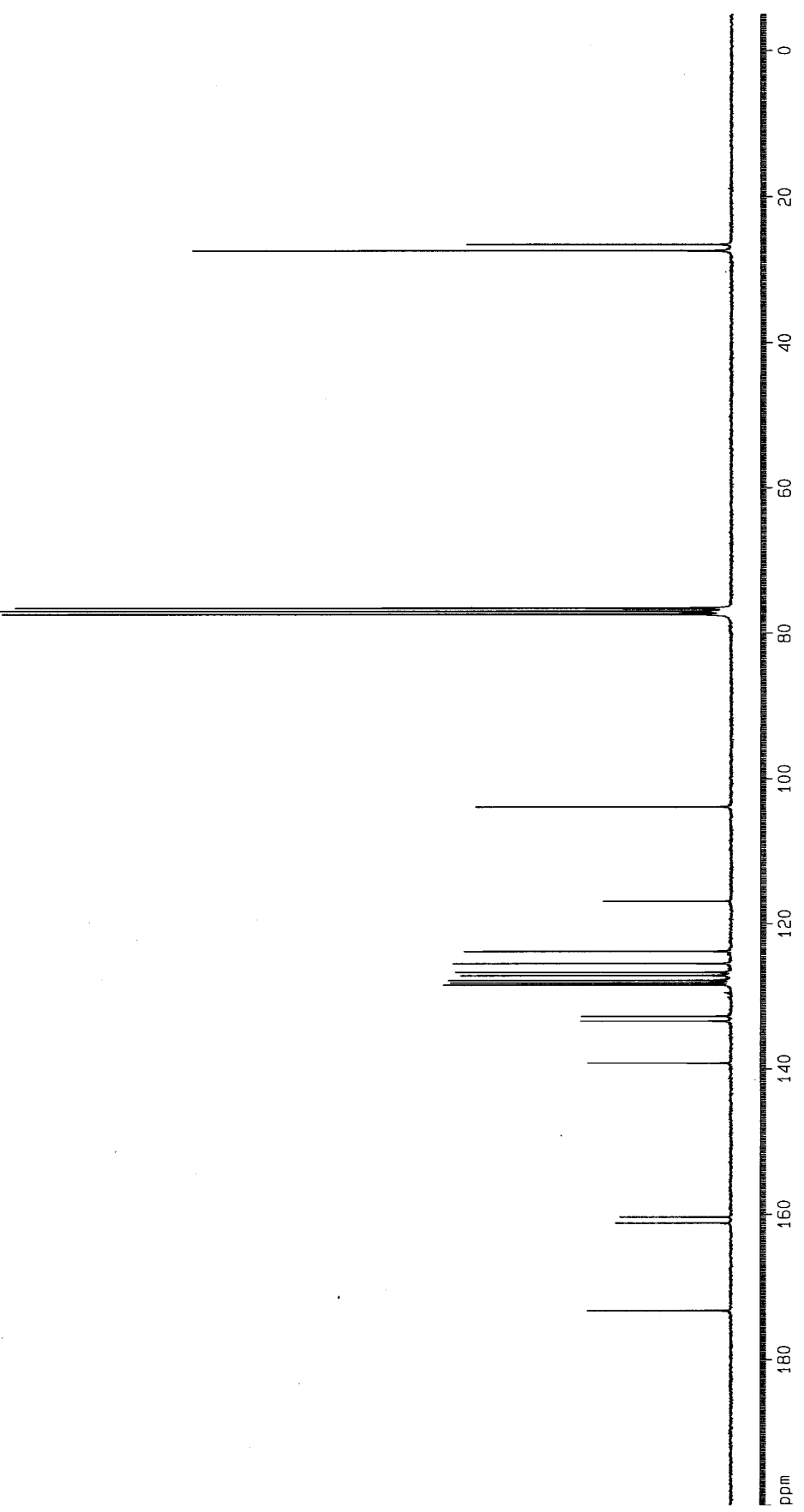

wdd

$\nabla$
$869^{\circ} 9 L$
2己 $9 L$

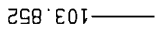

998.917

$\angle 9 L$ EटT

$\angle D D^{\circ} S C \downarrow-7$
$\square \angle 9 \cdot 9 己 \downarrow-7$

ट0I'LटT-

SEL L $\angle 2$

st०.85ा

IDL. टET-

टट० EEโ

IBI 6 โ

ISE O9I

SLI I I

$6 I C \cdot E L T=$

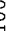

品

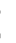

言 


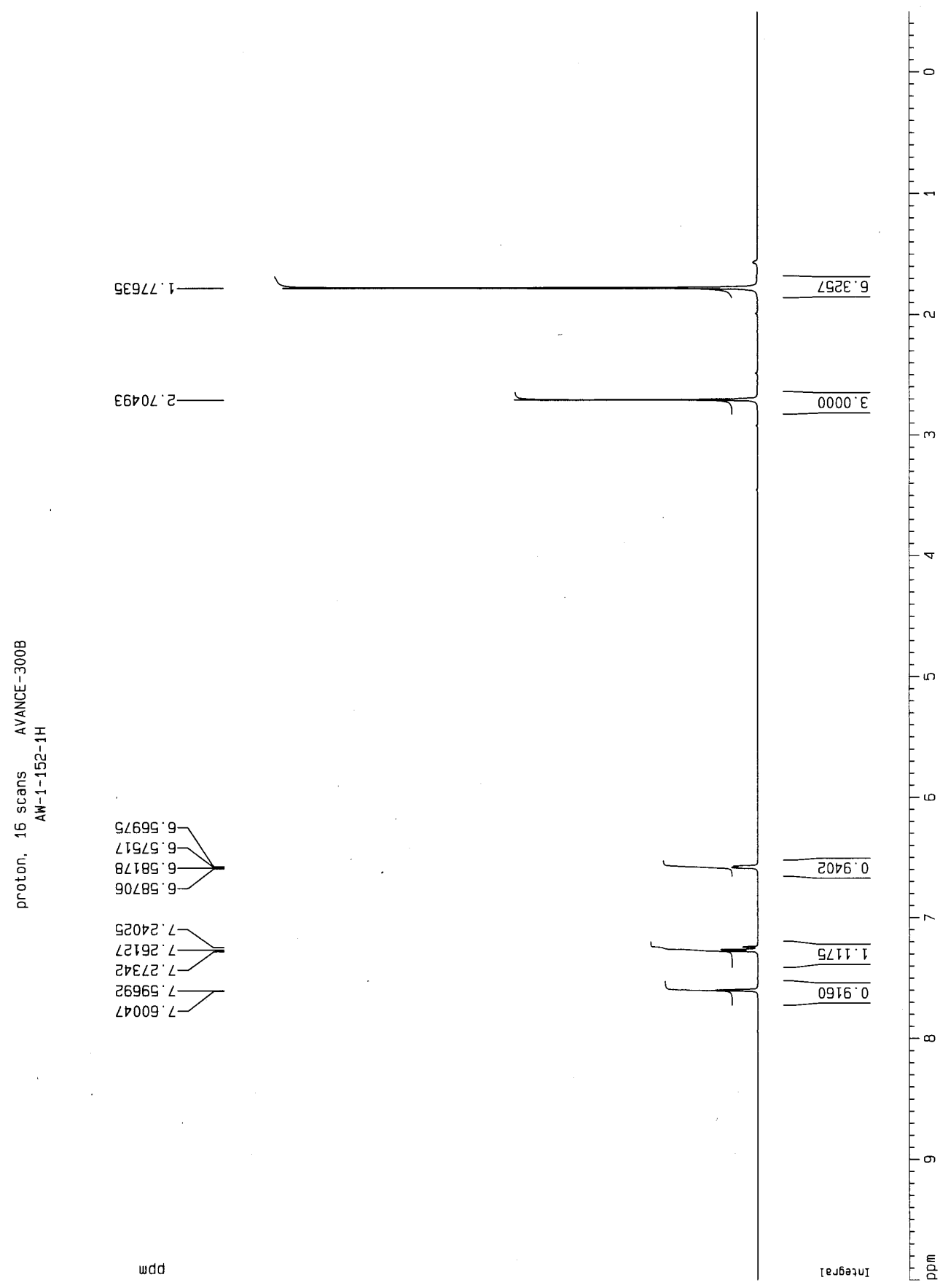




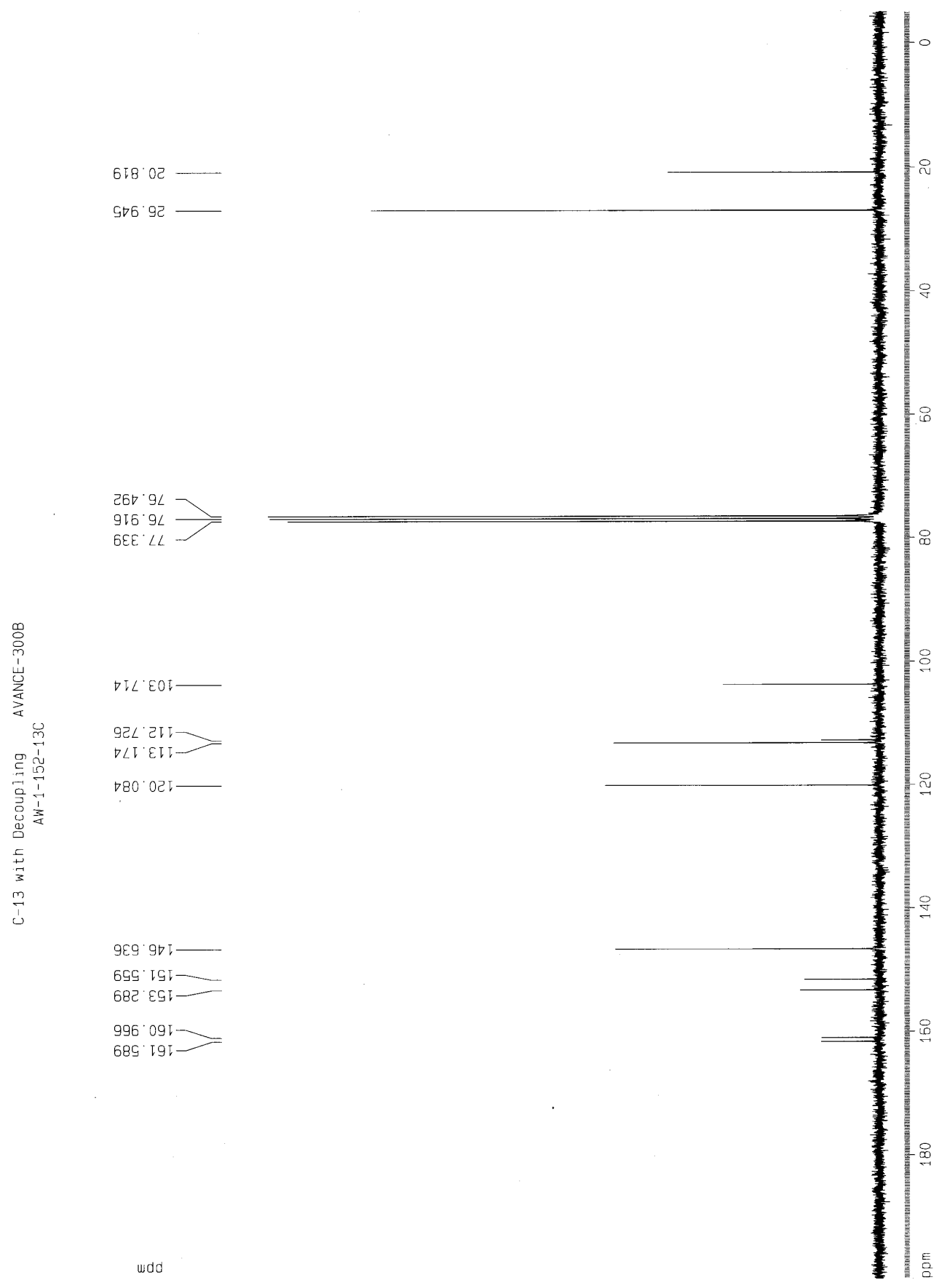




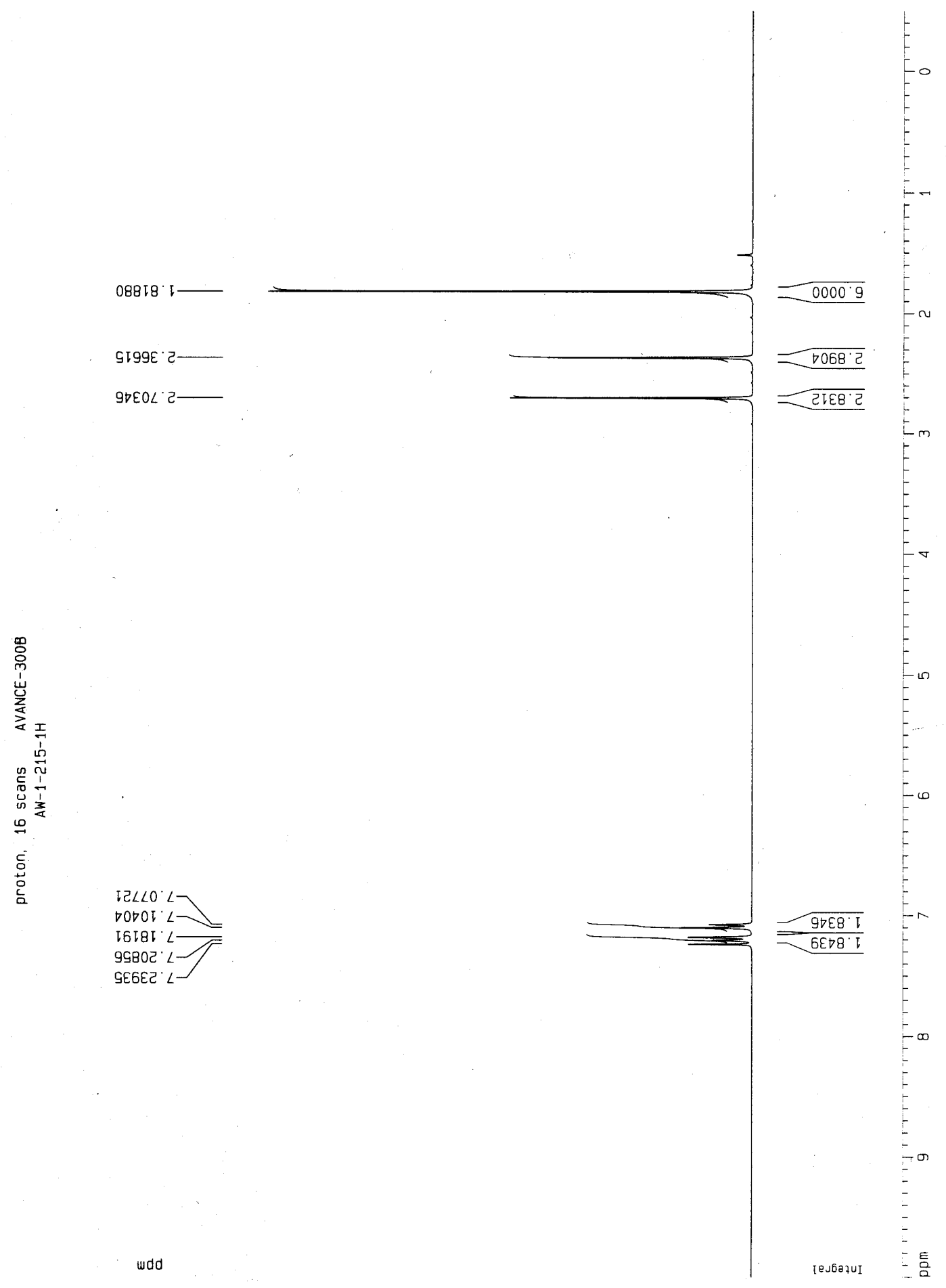




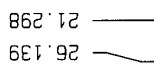

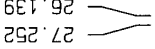

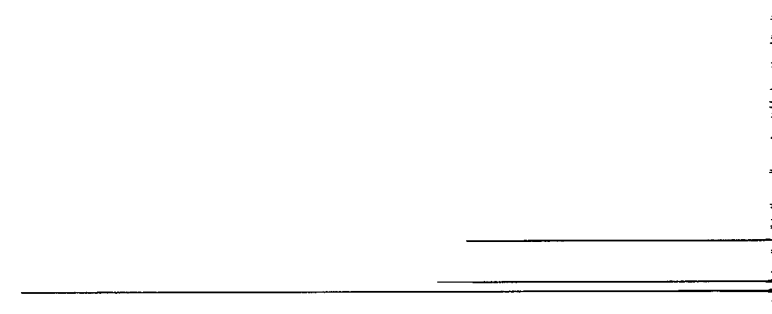

$99 \nabla^{\circ} \cdot 9 L$

$688^{\circ} 9 L$

$260^{\circ} \mathrm{LL}$

टIE ' $L L$

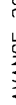

ILOEOL

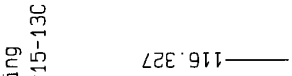

年

E0T'9己I- -

SI 6 6 I -

6I L BEI-
8I6 $6 E I-$

EIG $091-$
$061 \cdot 191=$

$69 \mathcal{C}^{\circ} E \angle T-$
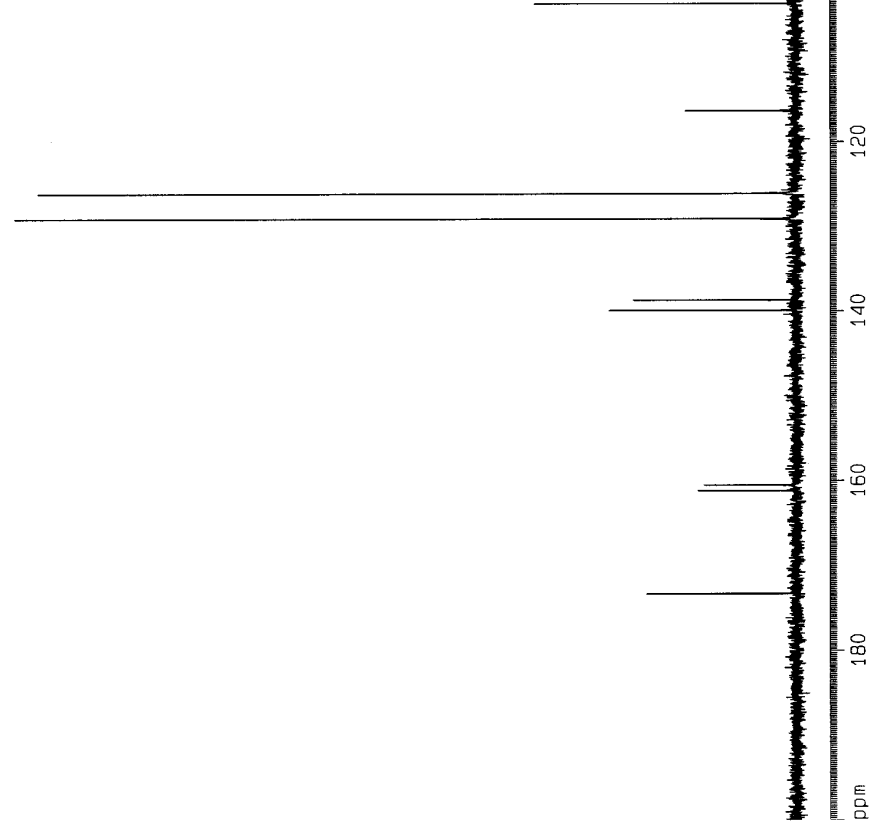


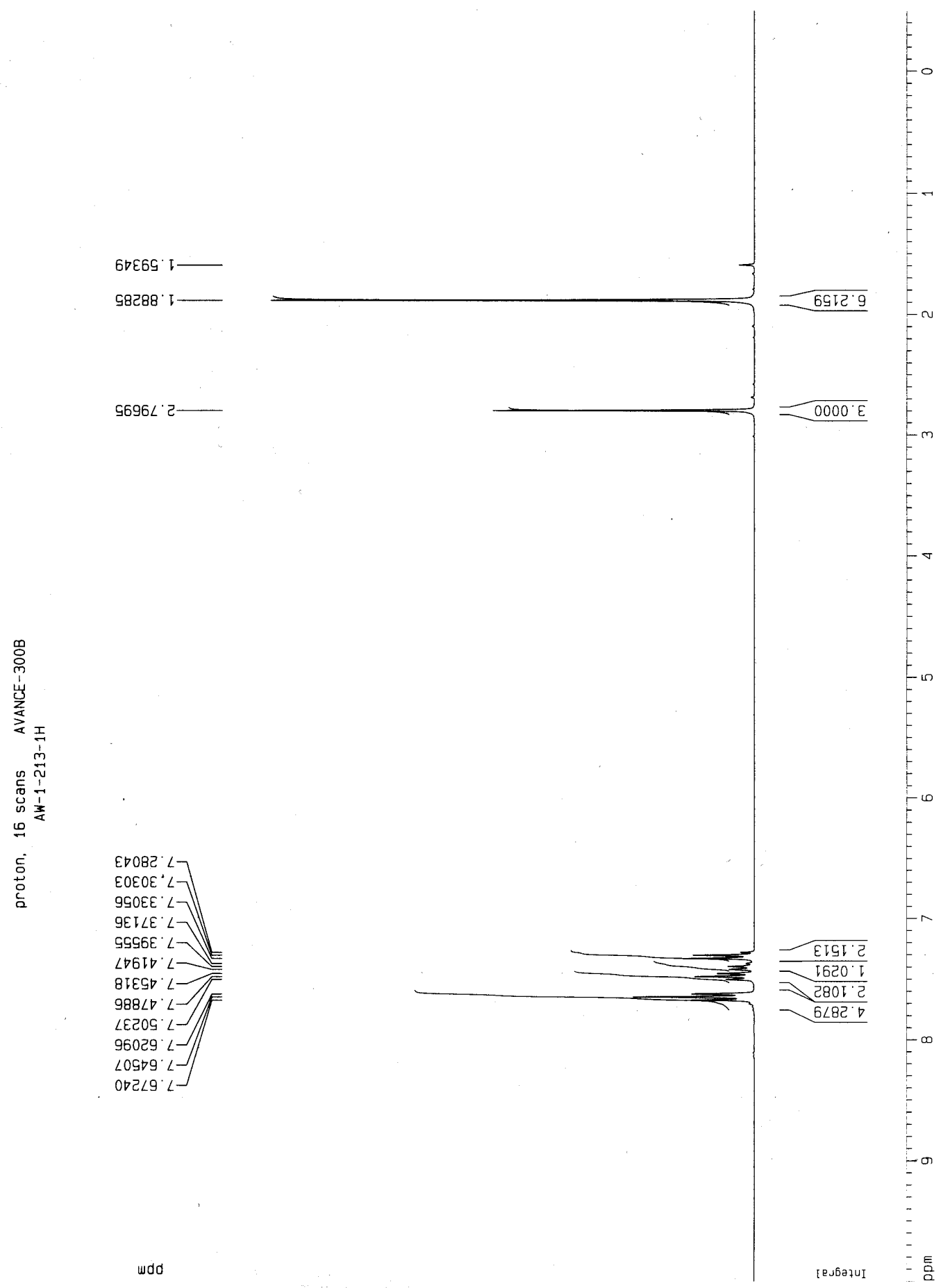




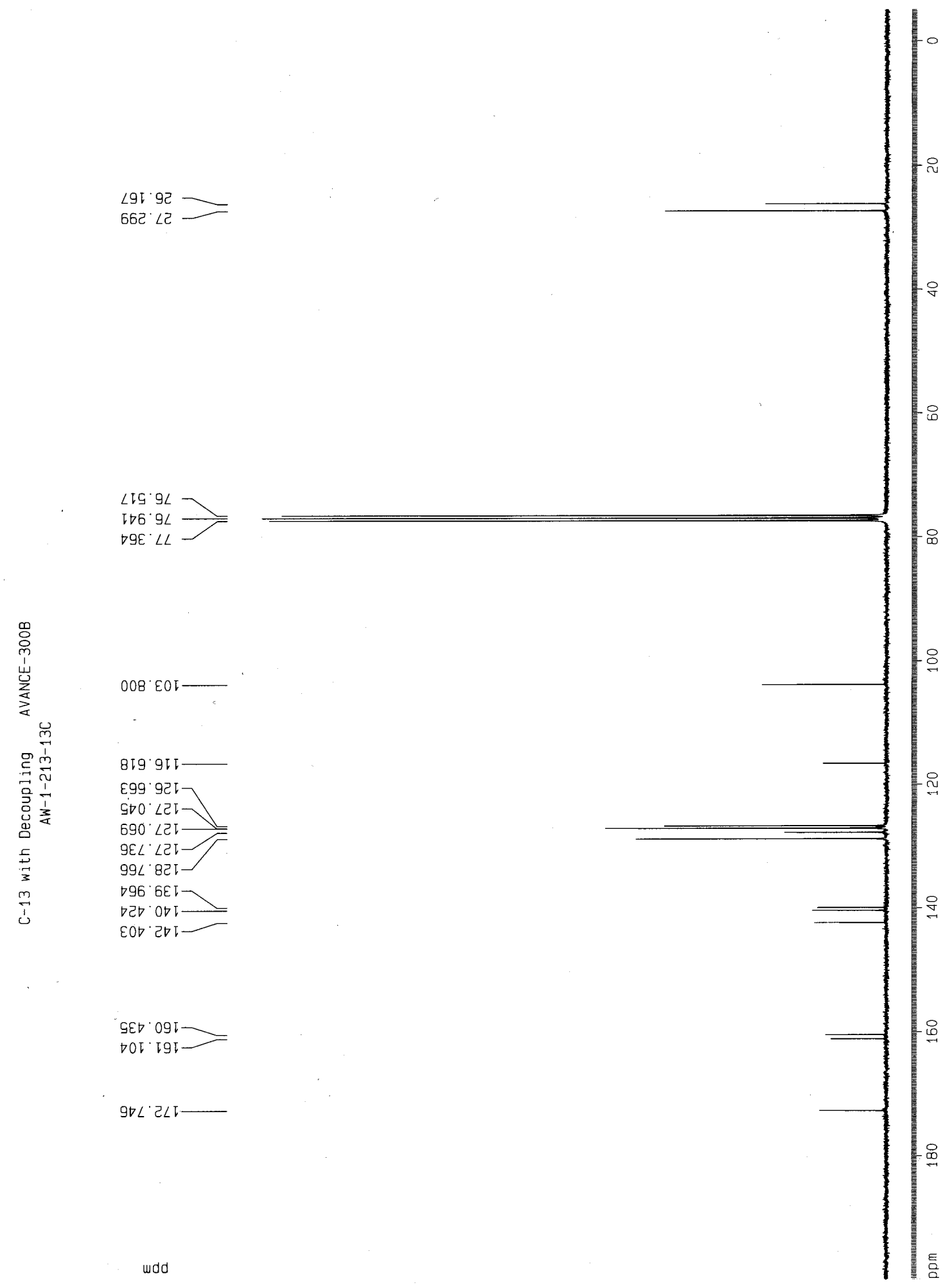




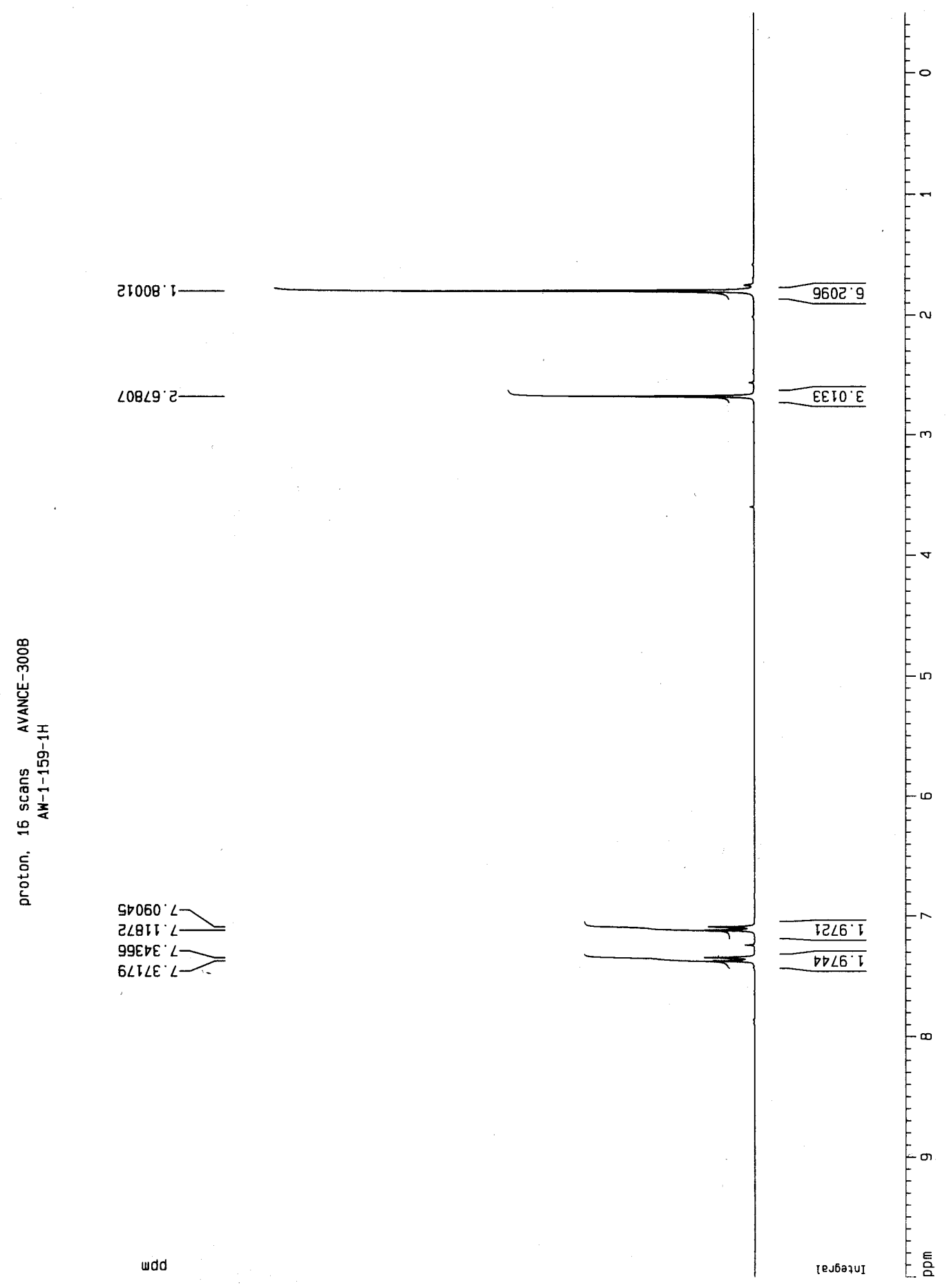




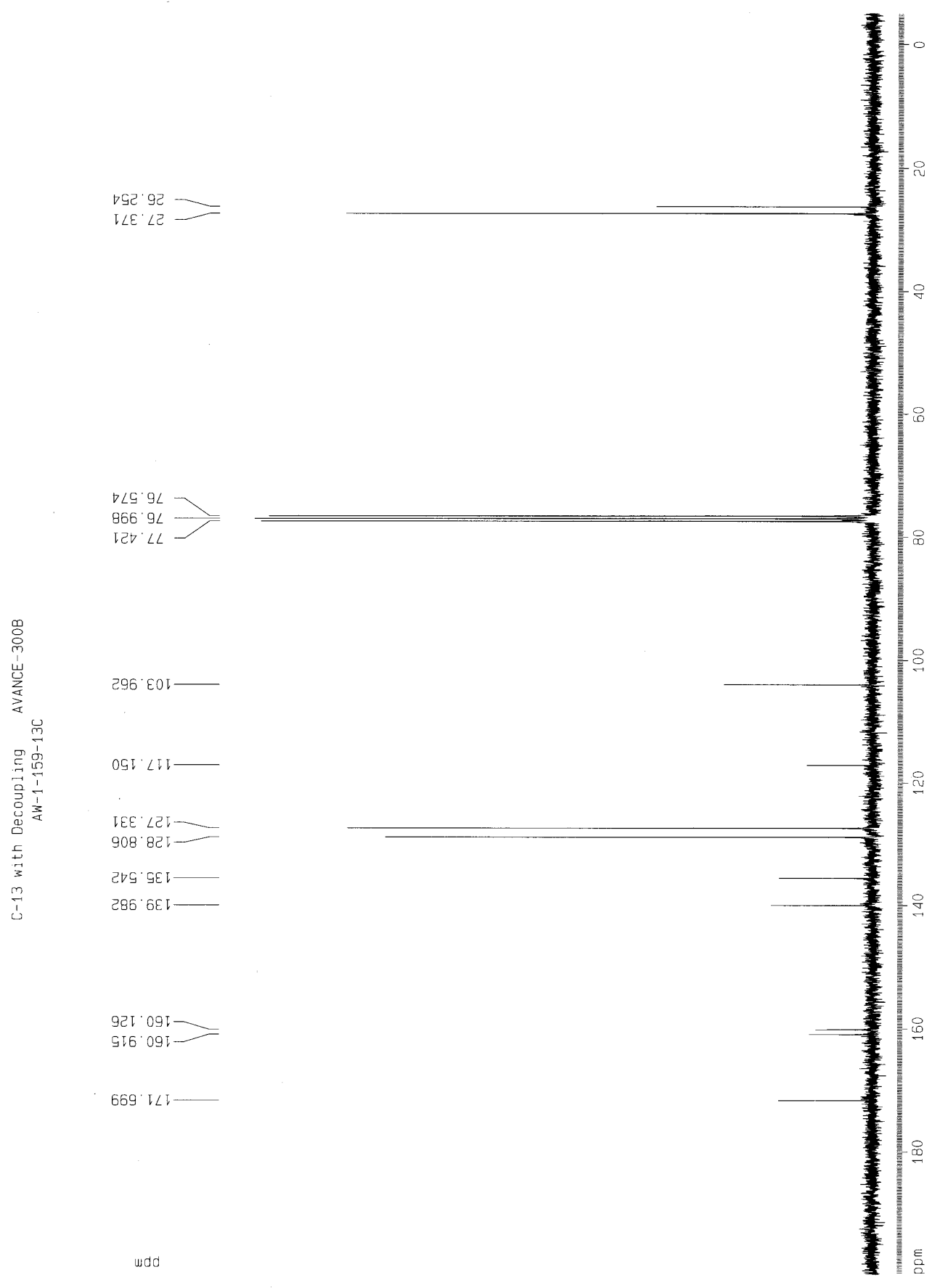


5-[1-(4-Bromophenyl)ethylidene]-2,2-dimethyl-1,3-dioxane-4,6-dione (1g)

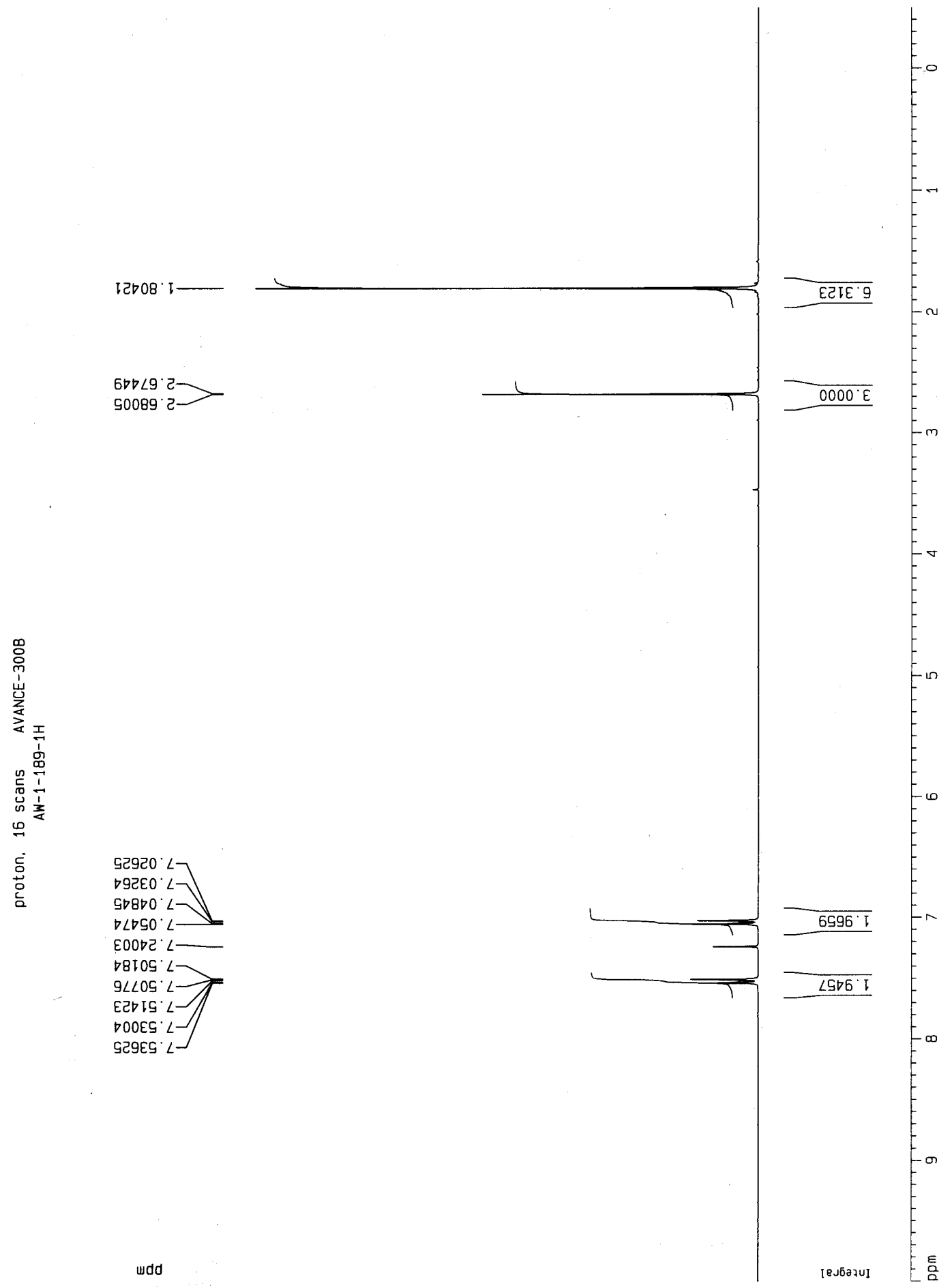


5-[1-(4-Bromophenyl)ethylidene]-2,2-dimethyl-1,3-dioxane-4,6-dione (1g)

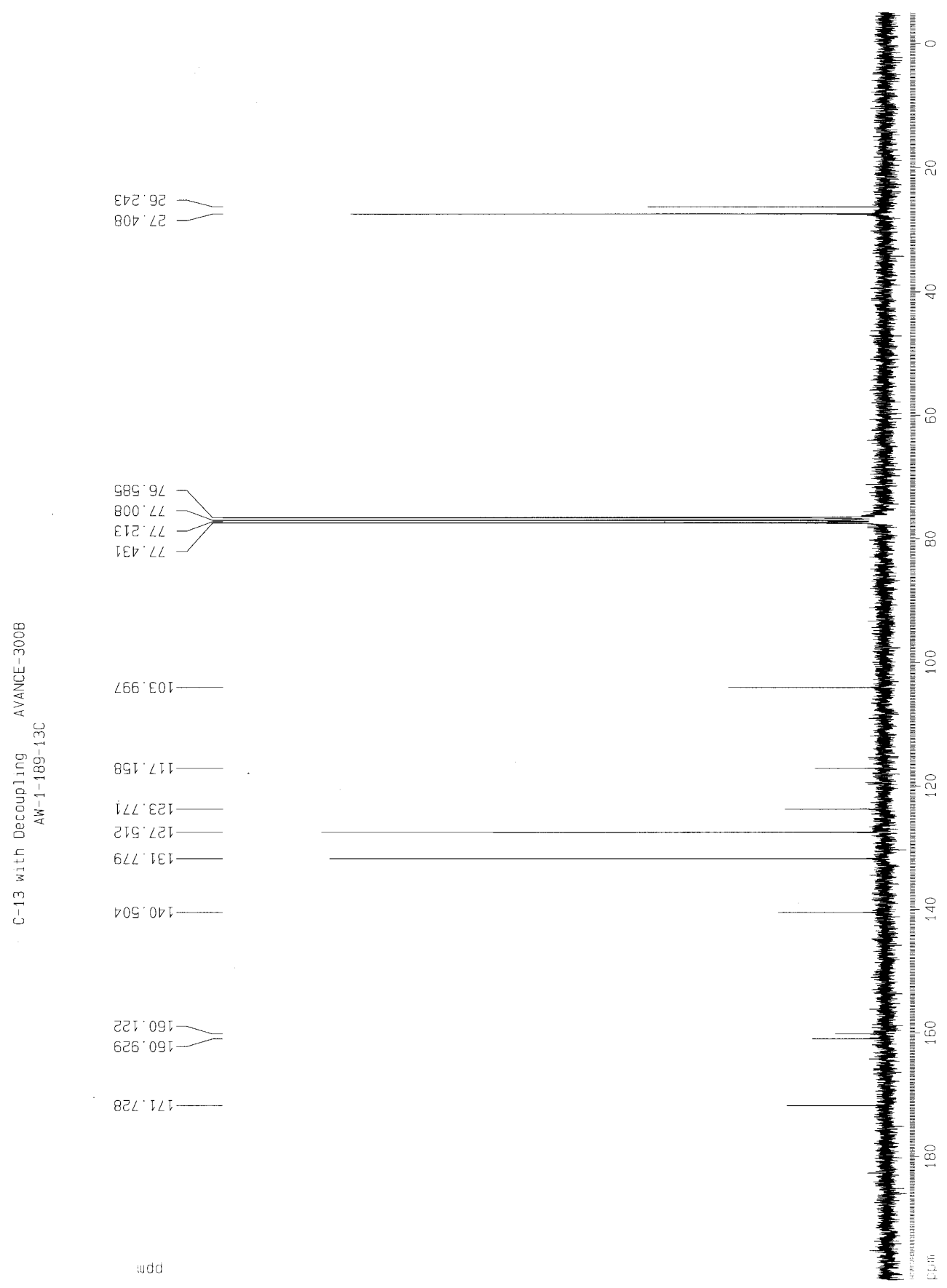




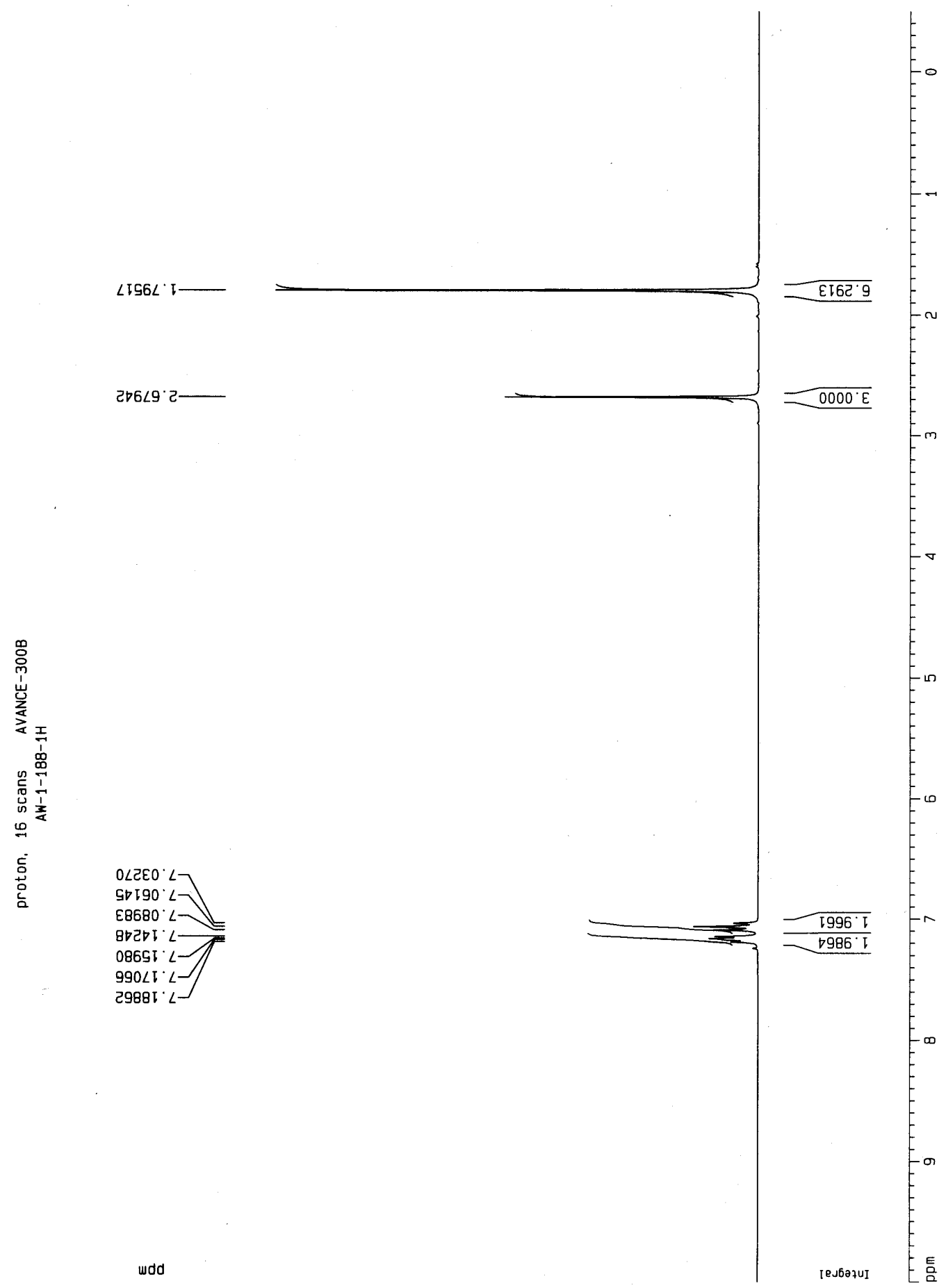




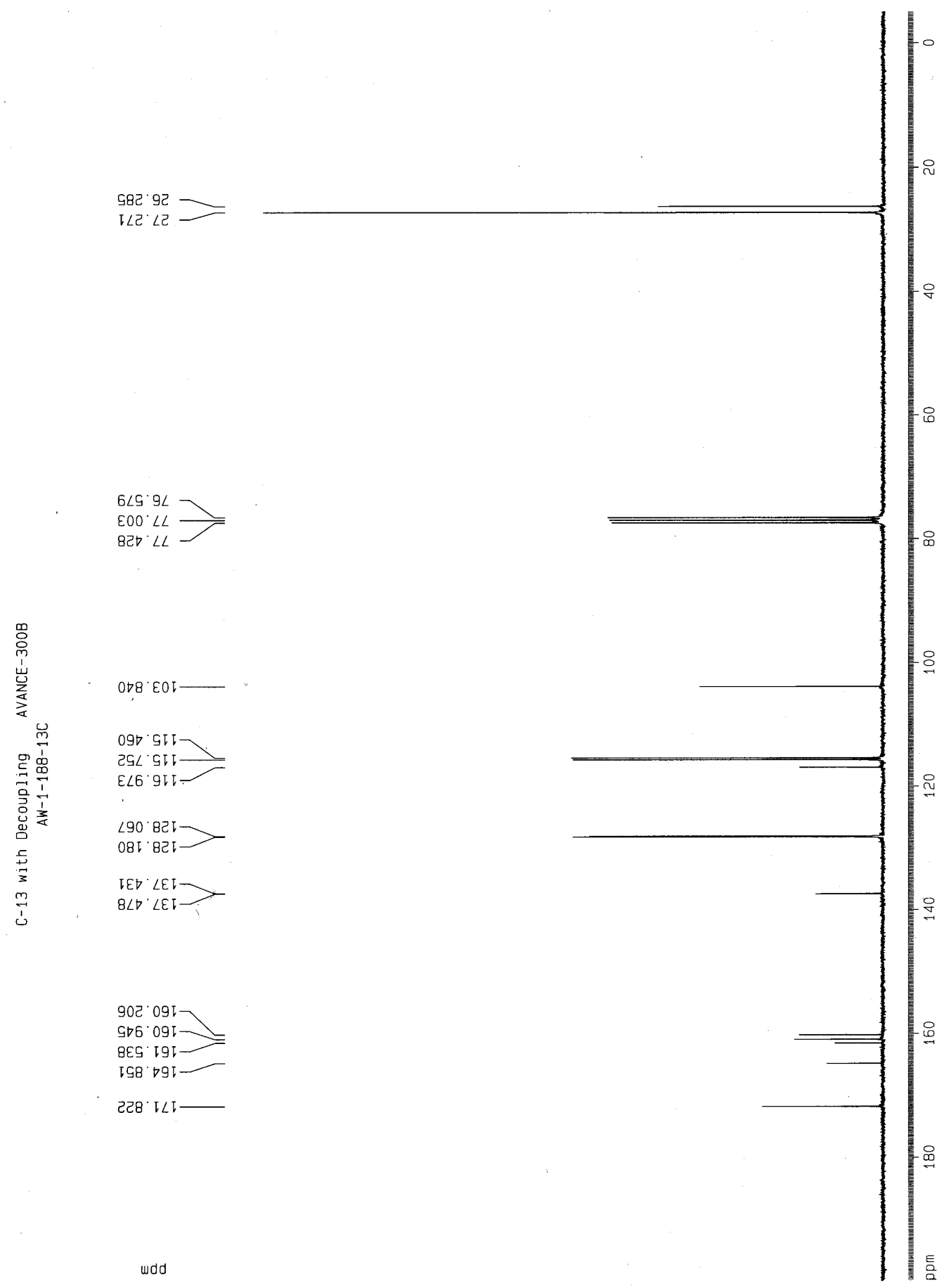




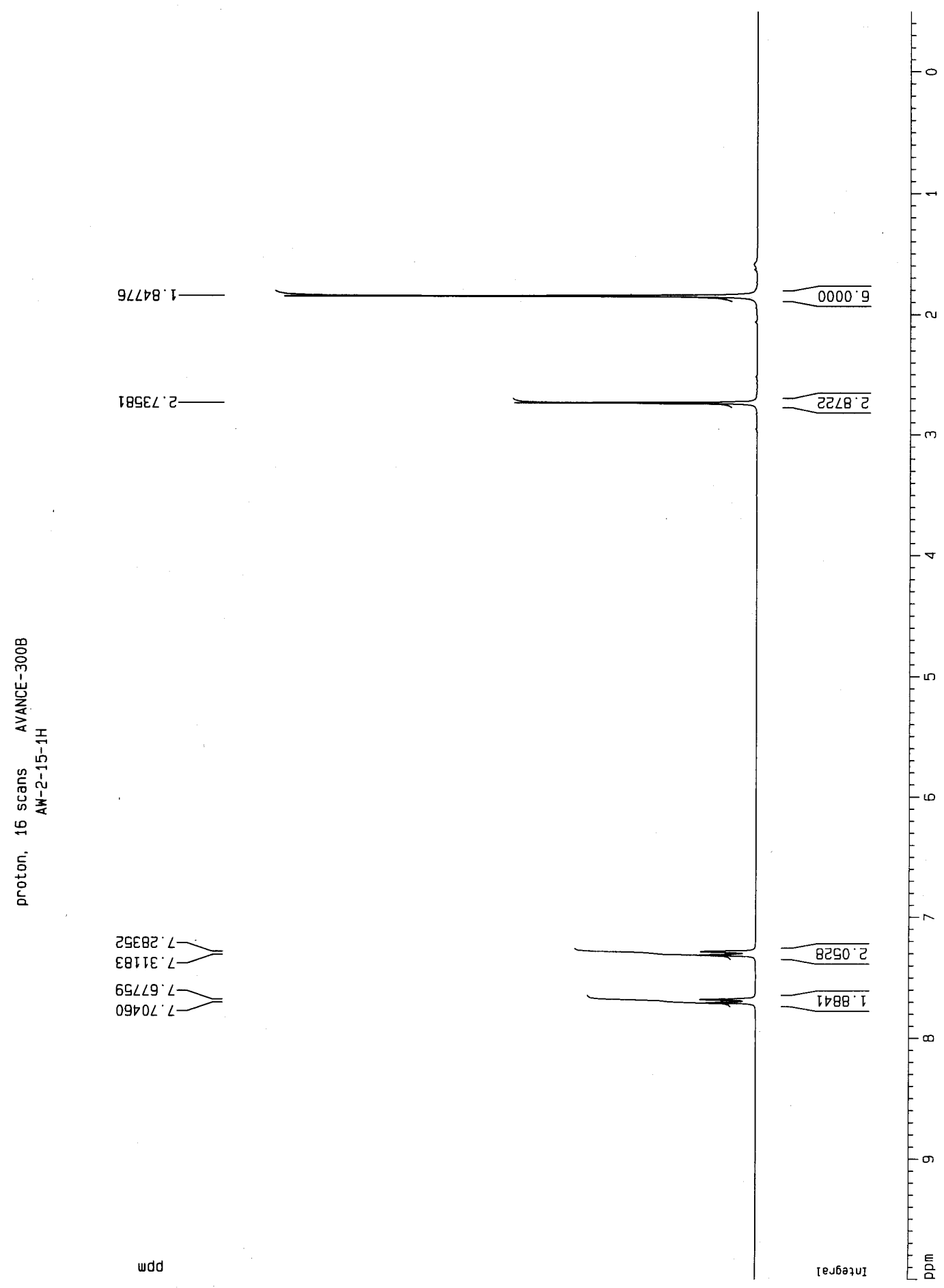




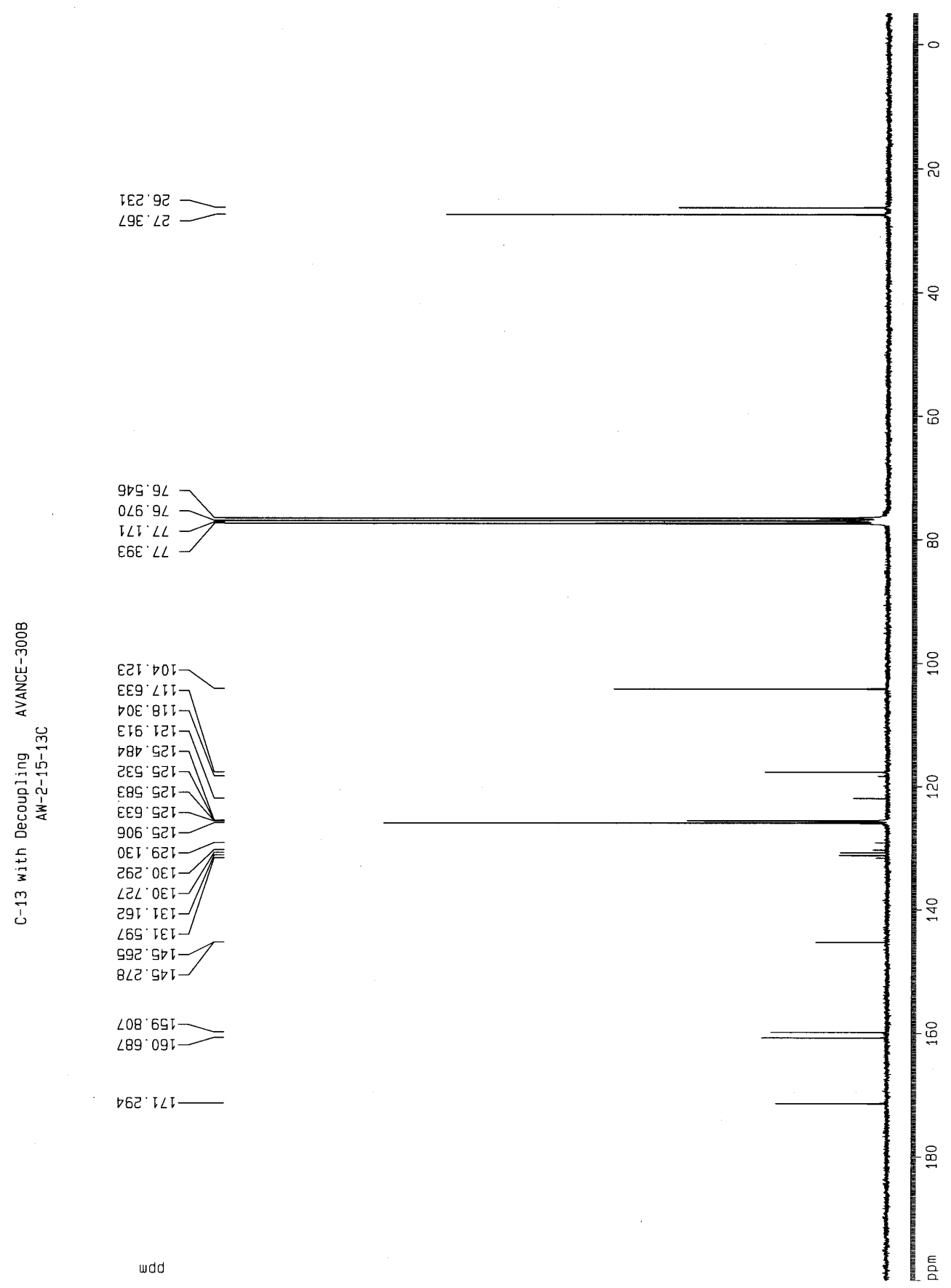




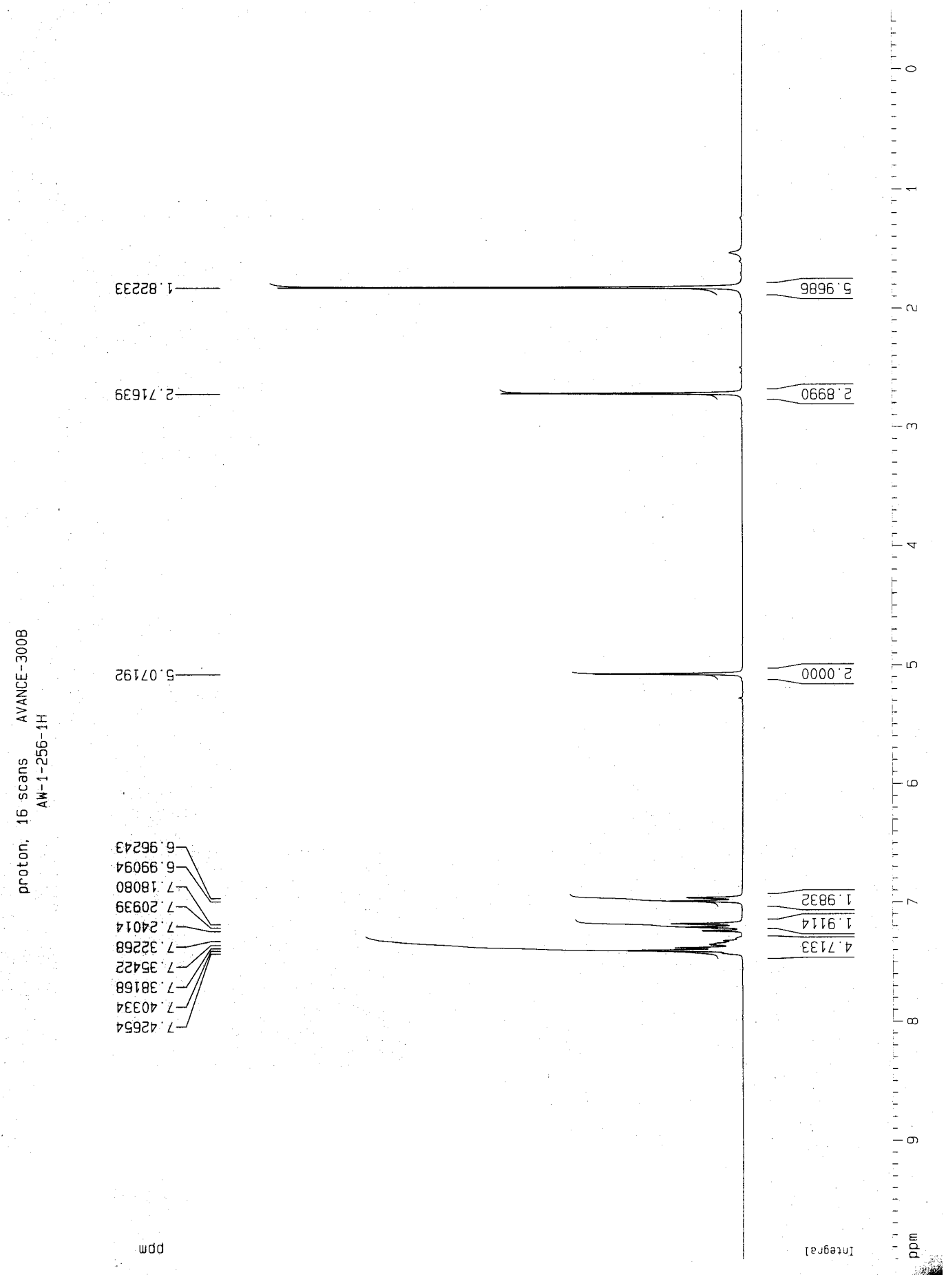


5-1-[4-(Benzyloxy)phenyl)]ethylidene-2,2-dimethyl-1,3-dioxane-4,6-dione (1j)
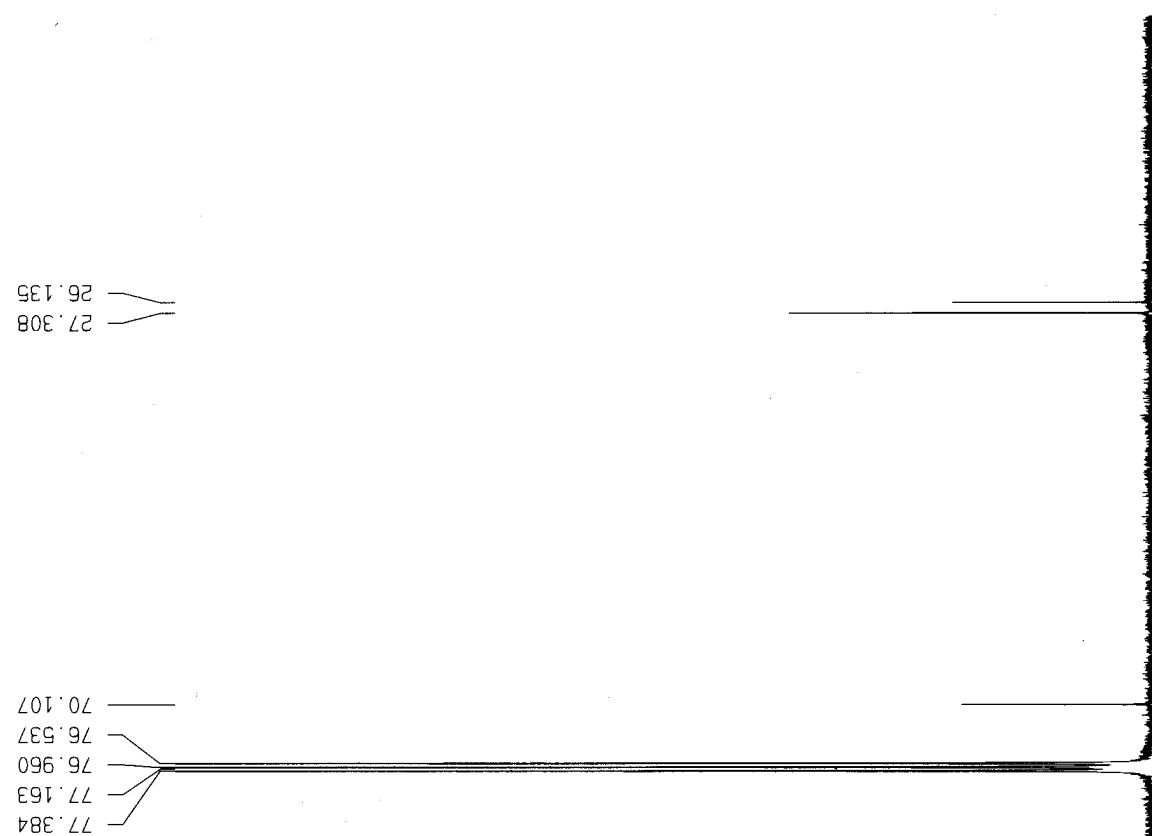

$\angle E 9^{\circ}$ EOL
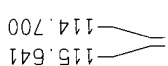

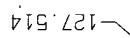

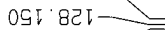

OEg.851-

ट88: $E$ E

$O D E^{\prime} 9 E I \longrightarrow$

876.091

$9 \nabla E_{191-}$

998 CLI

wdd

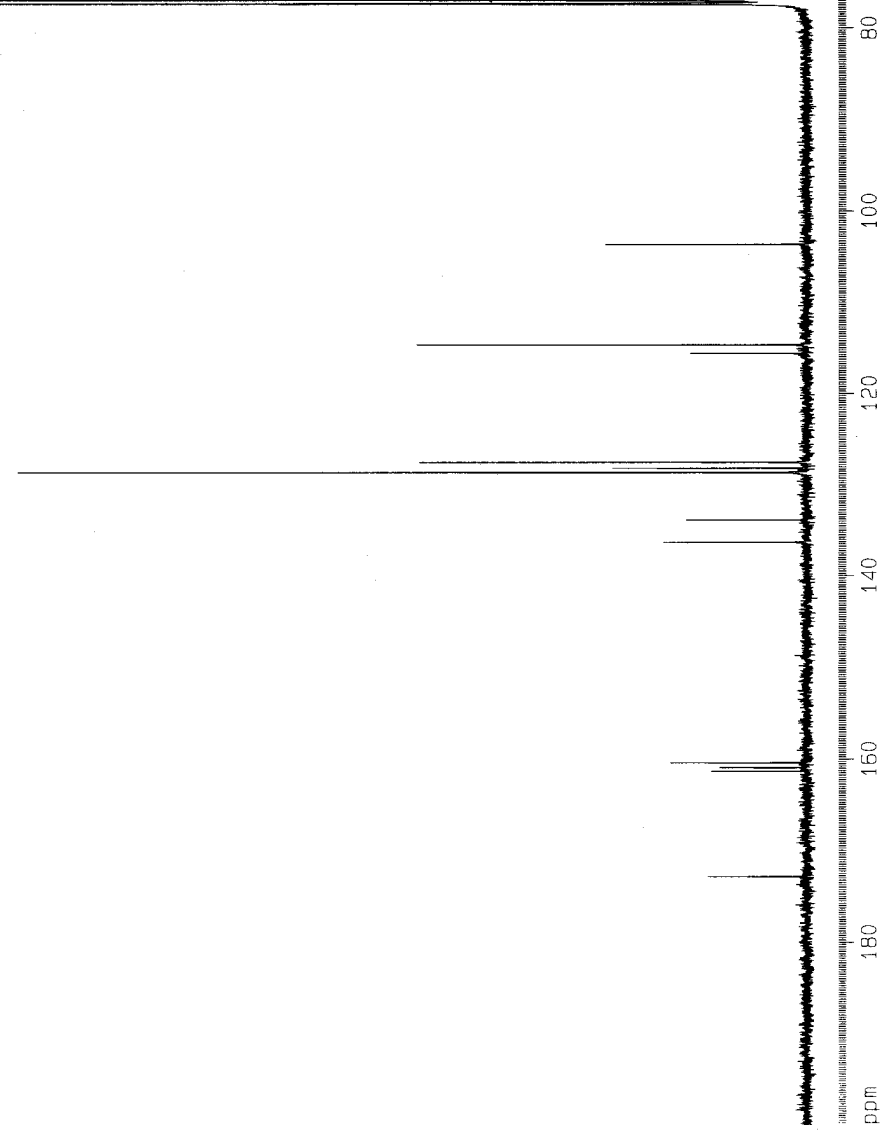




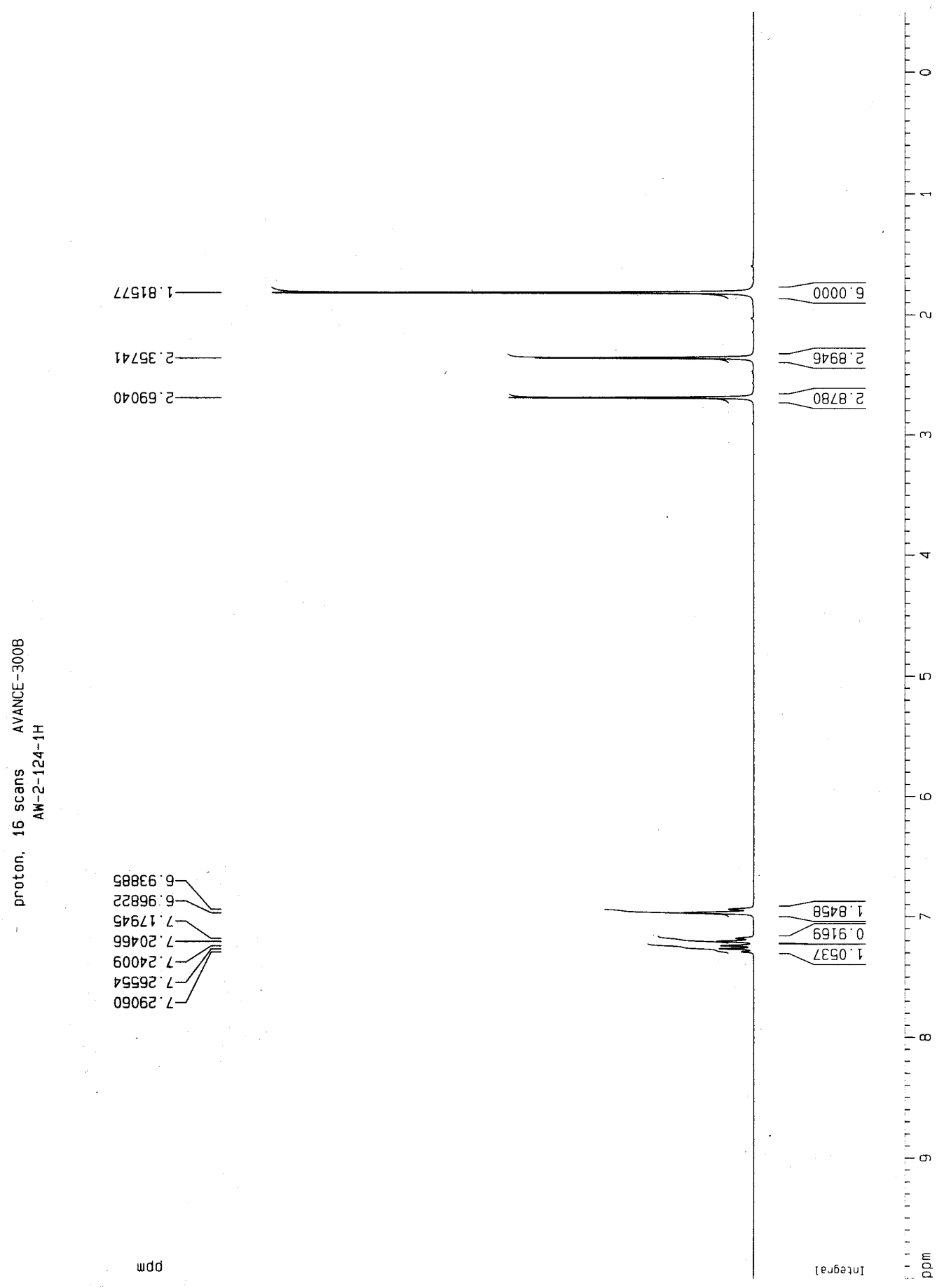




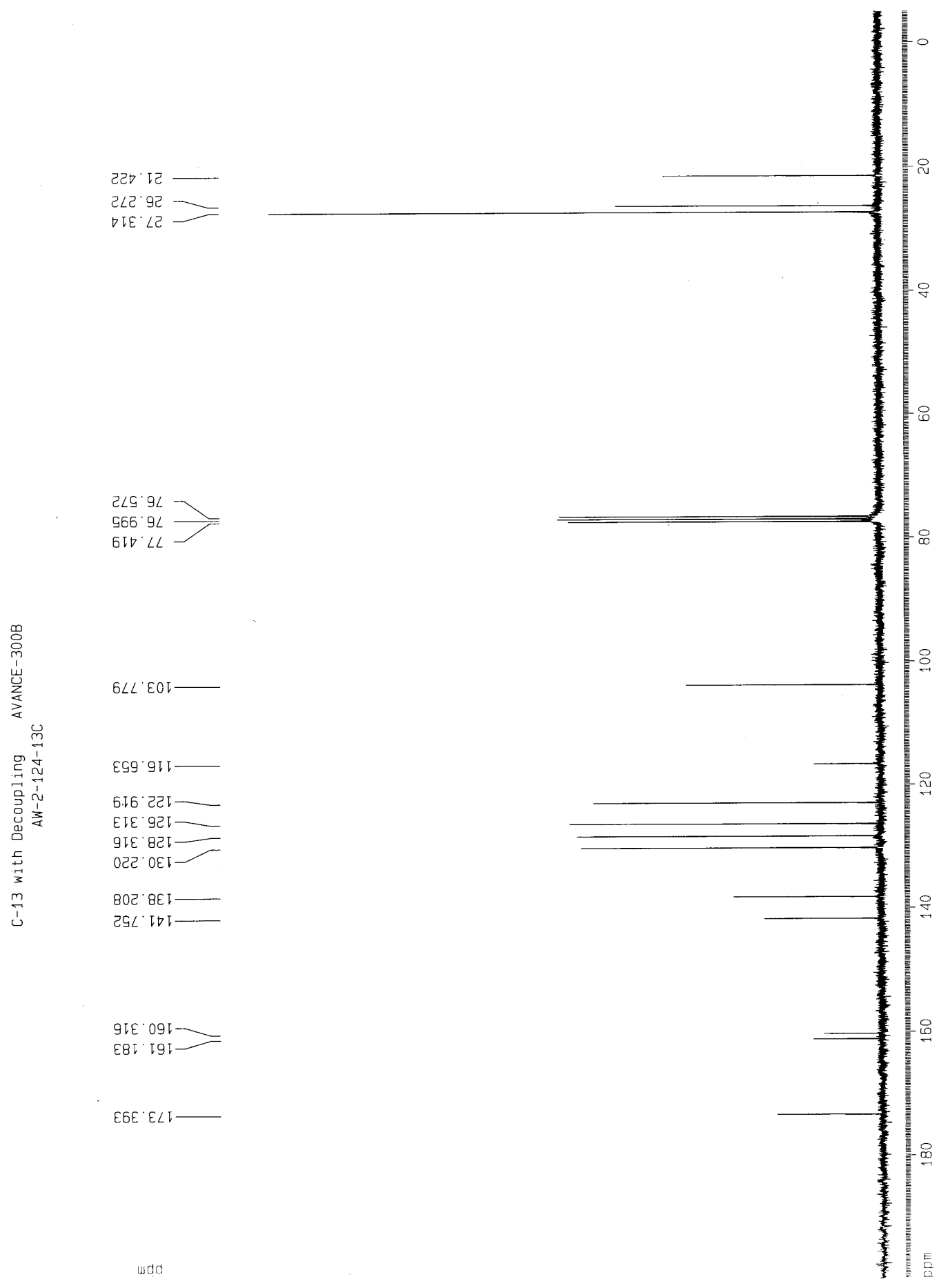




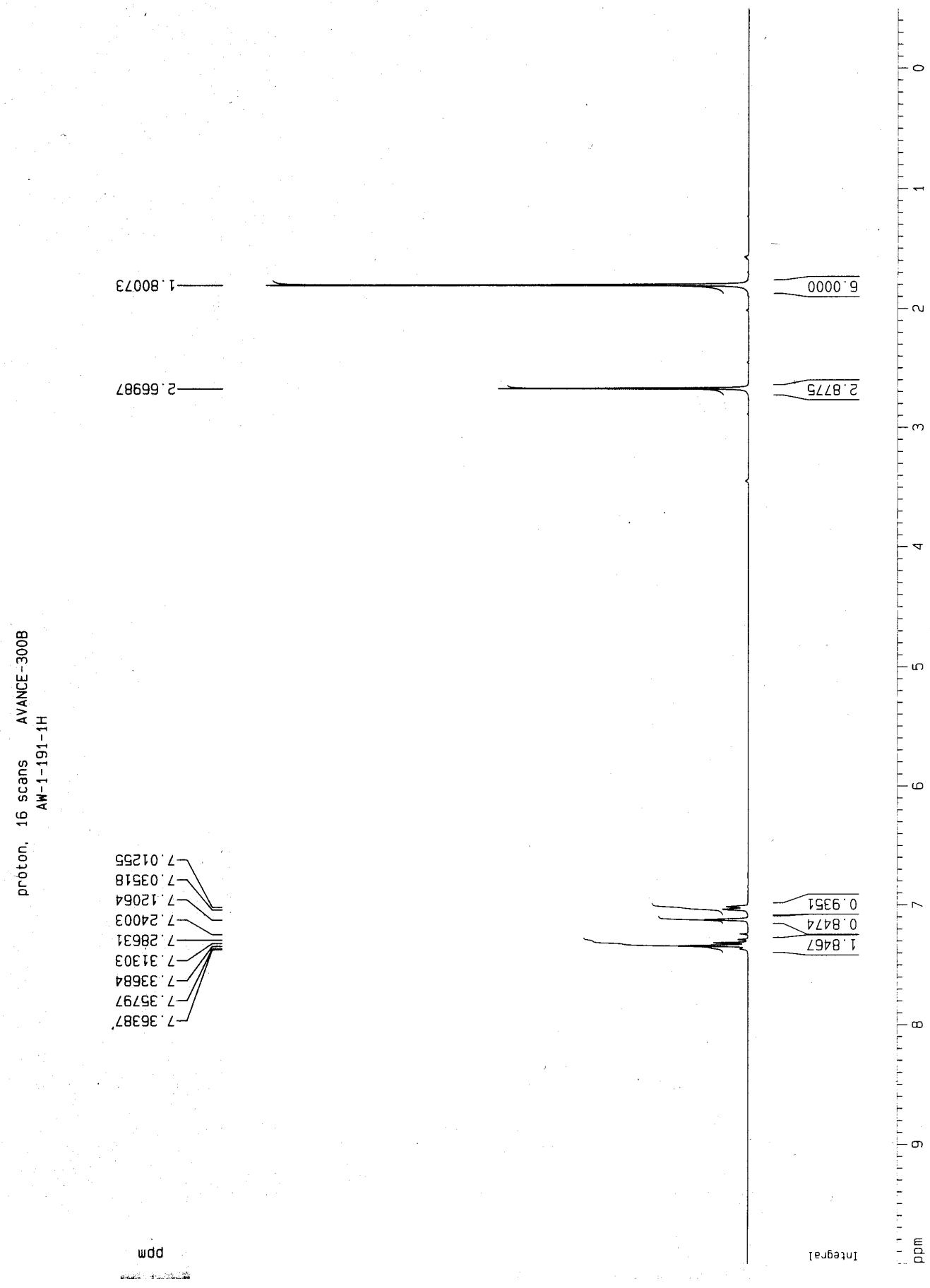




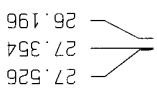

$6 \angle 9^{\circ} 9 \angle$

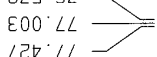

$220 \circ 01 \cdots$

9
8
0
4
岁
$\sum_{1}$

z

EOT $\angle L T$

$898^{\circ} \varepsilon 己$.

$029.95 T$

$\angle 81.621-=$

$508^{\circ} 625$

OEt $\nabla \varepsilon \digamma$

GIE.EDI

$578.691-$

$92 T^{\circ} T \angle L$

wdd

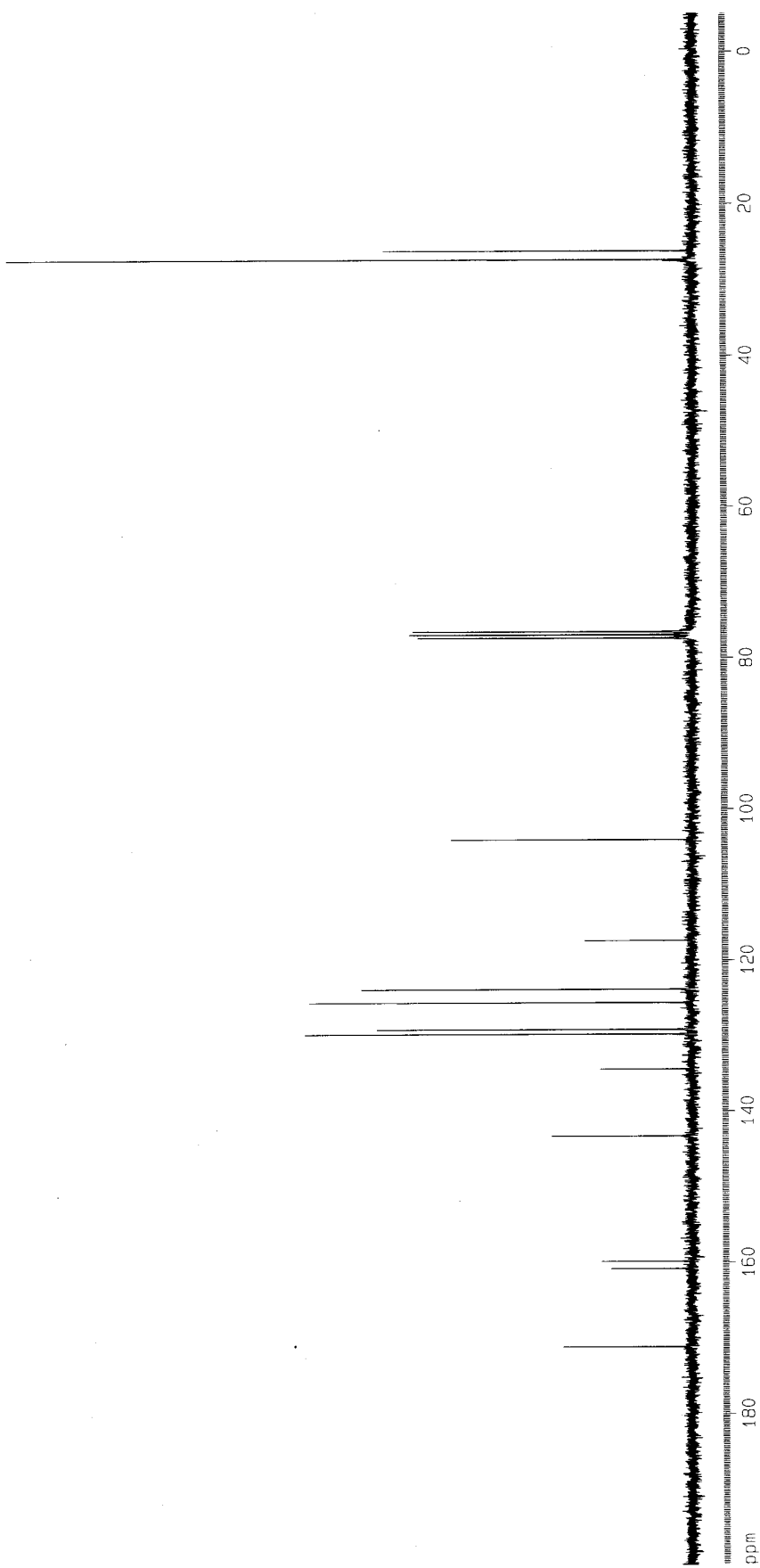




\section{5-1-[3-(Benzyloxy)phenyl)]ethylidene-2,2-dimethyl-1,3-dioxane-4,6-dione (1m)}

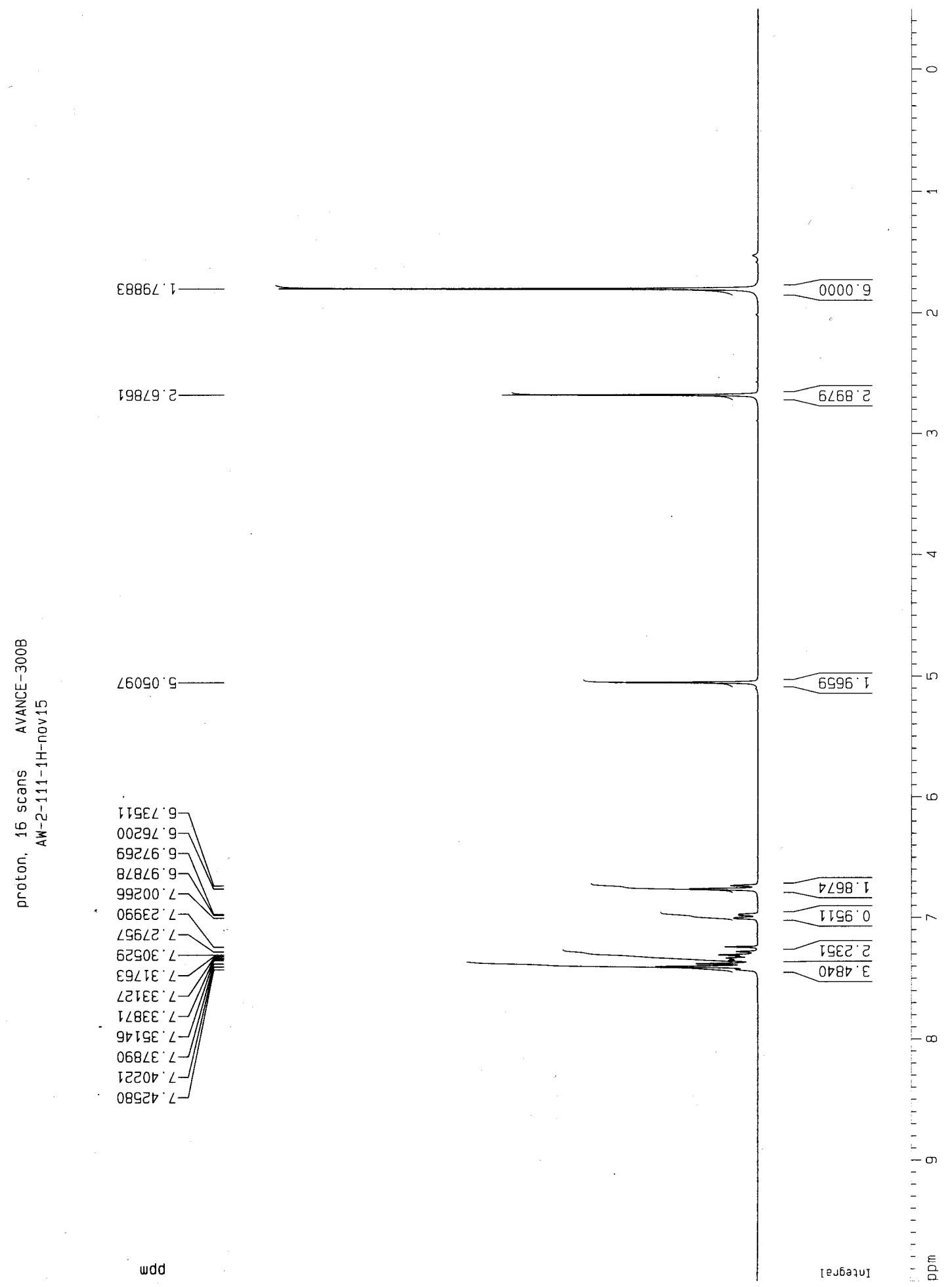



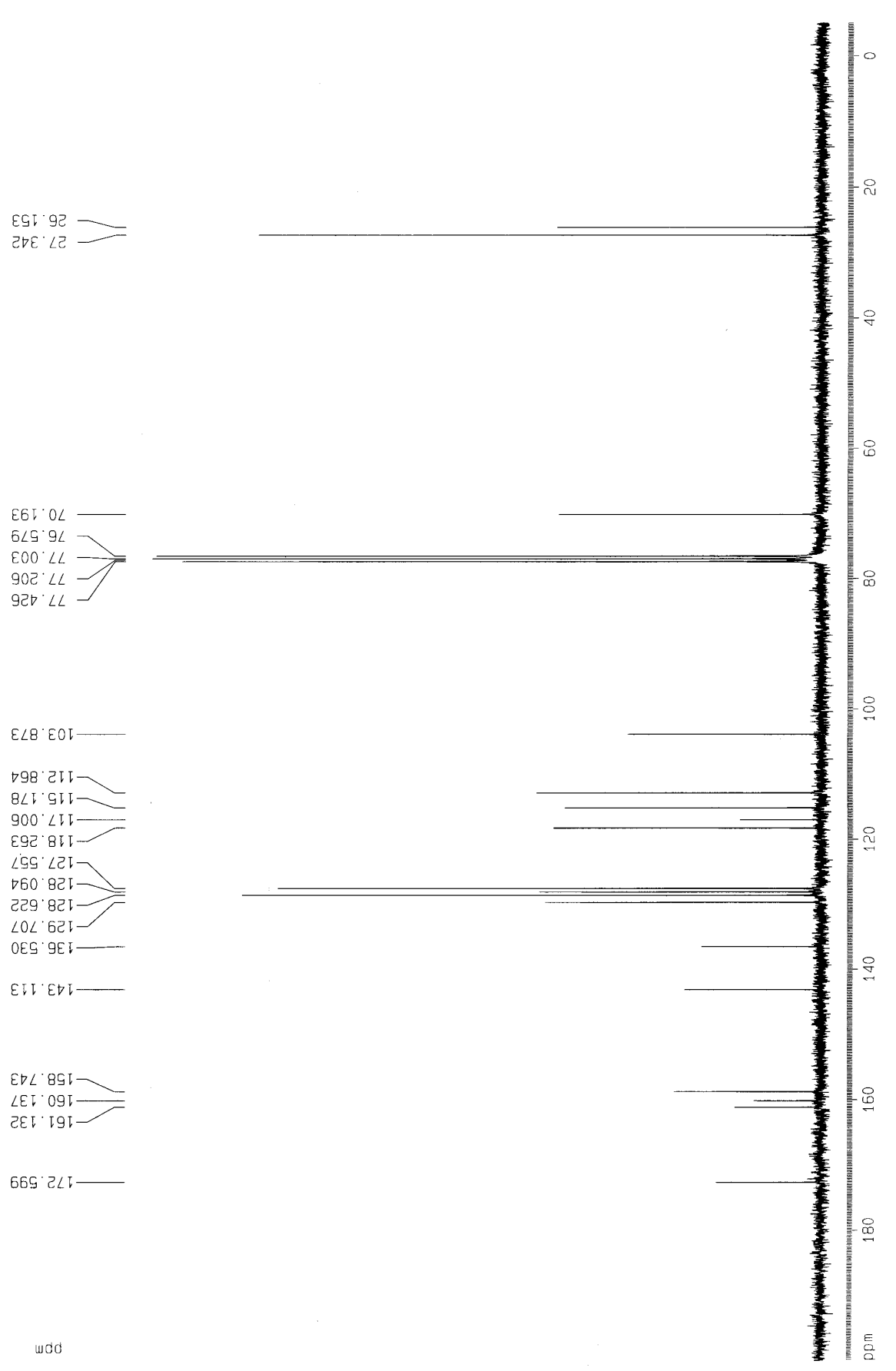

$\varepsilon \angle 8^{\circ} E O \amalg \ldots$

$\nabla 98^{\circ}$ 己几一

8LI. GI -

$900^{\circ} \angle I V$

Ege. 8 it

$\angle 9 G^{\circ} \angle 2 I \longrightarrow$

$\triangle 60^{\circ} 8 \mathrm{CL} \longrightarrow$

ट29.8टा

EITEDI-

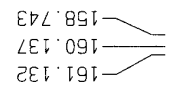

$669 \cdot 2 \angle L$

wdd 


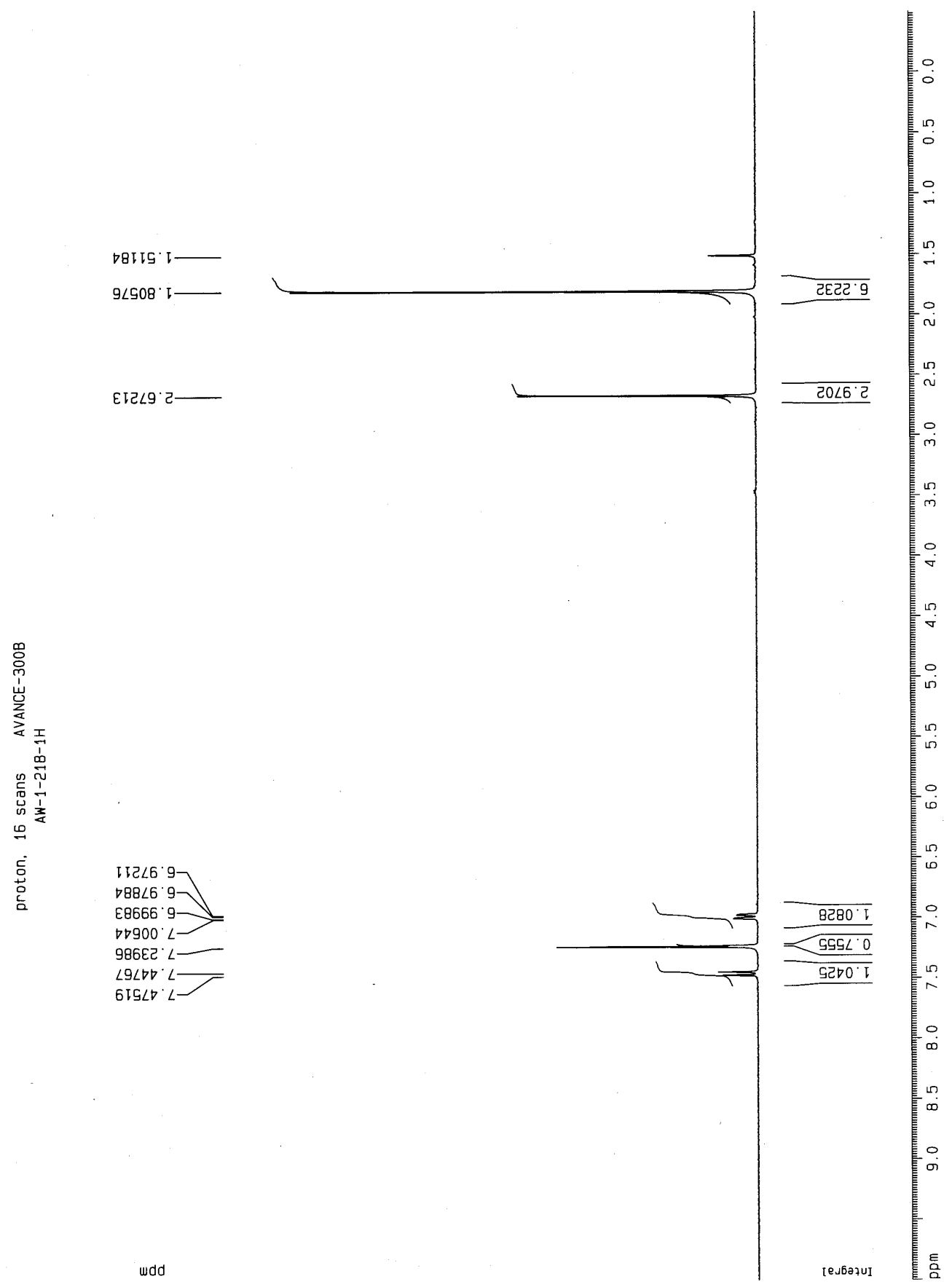




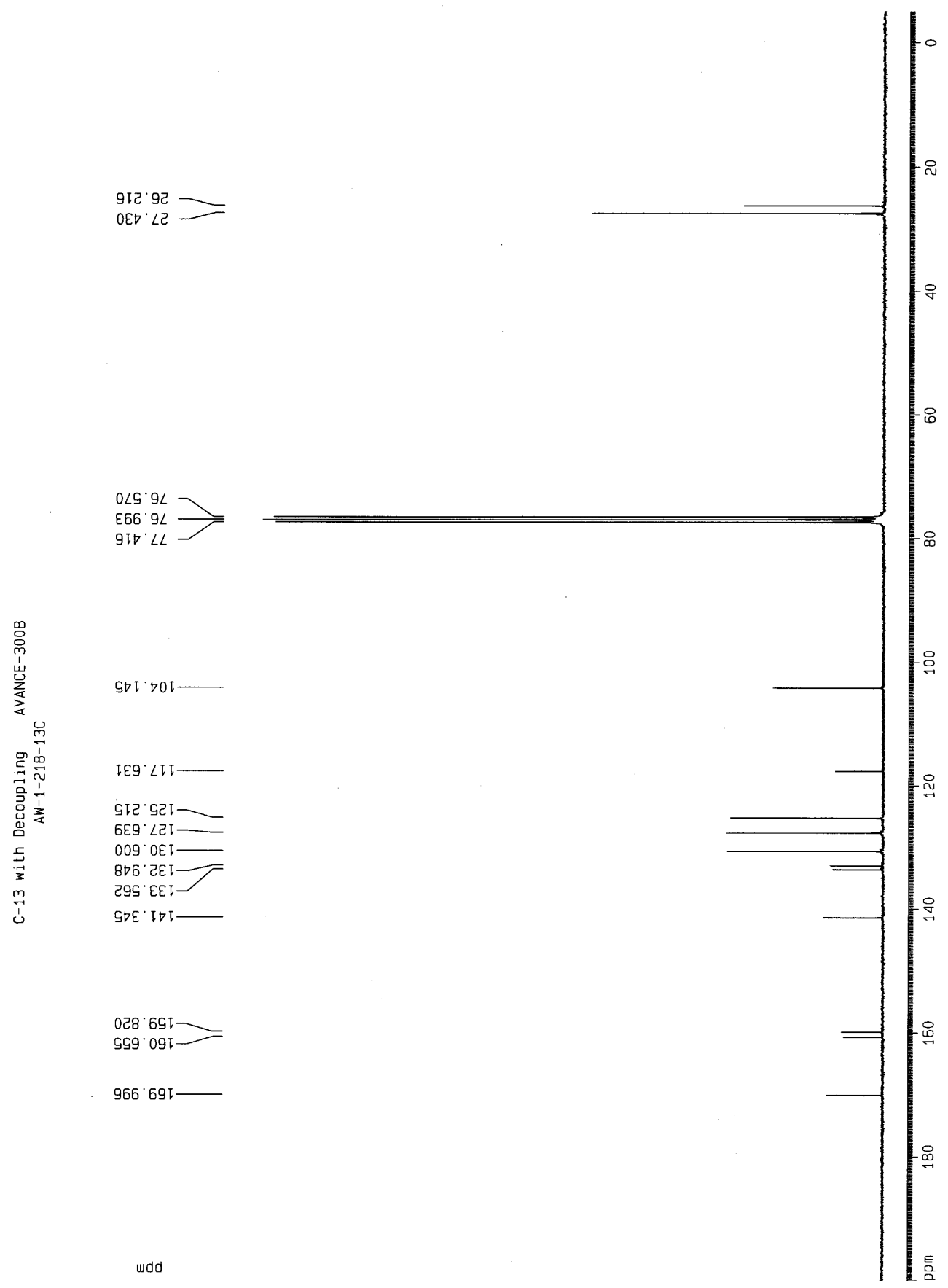




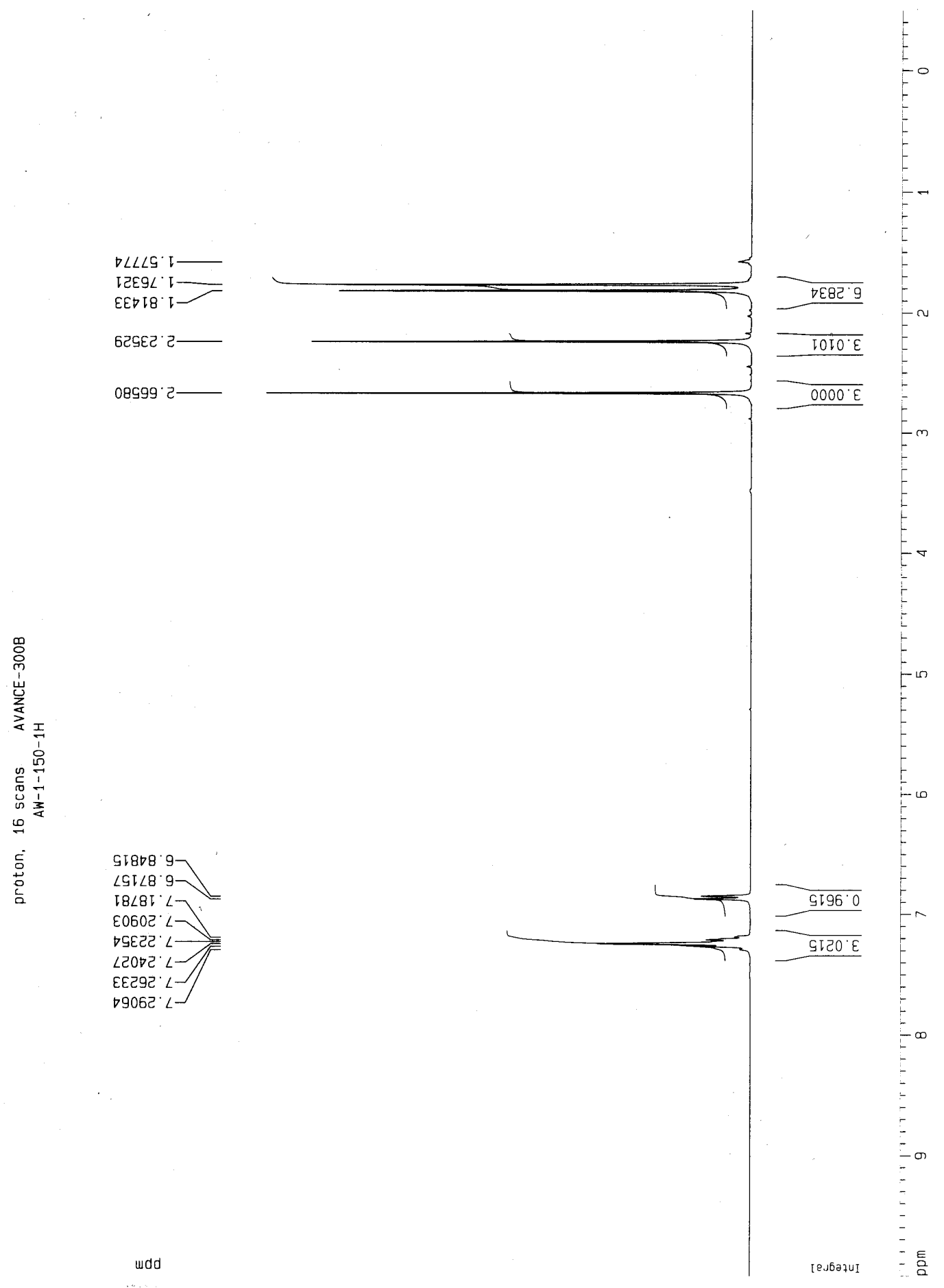




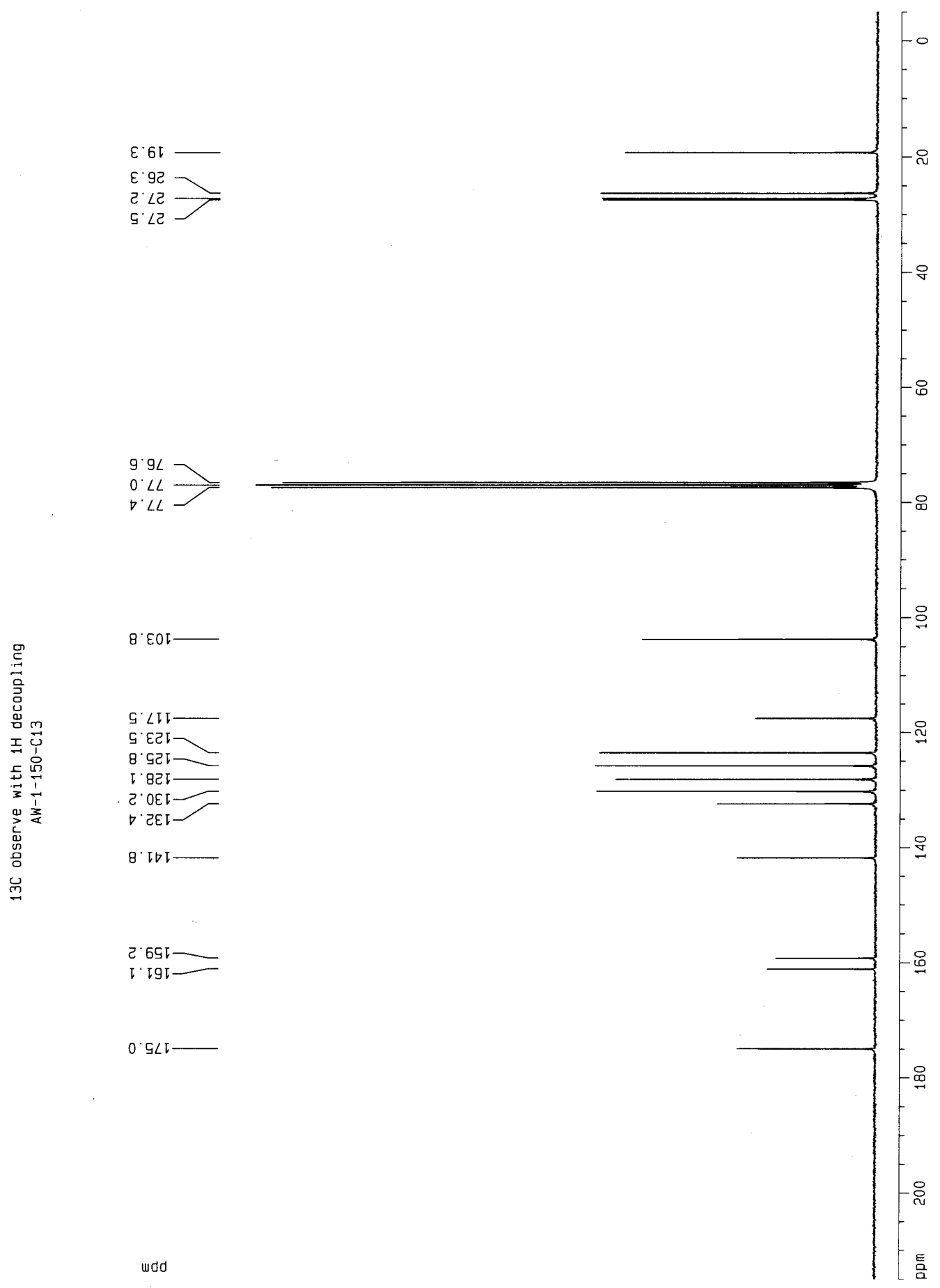




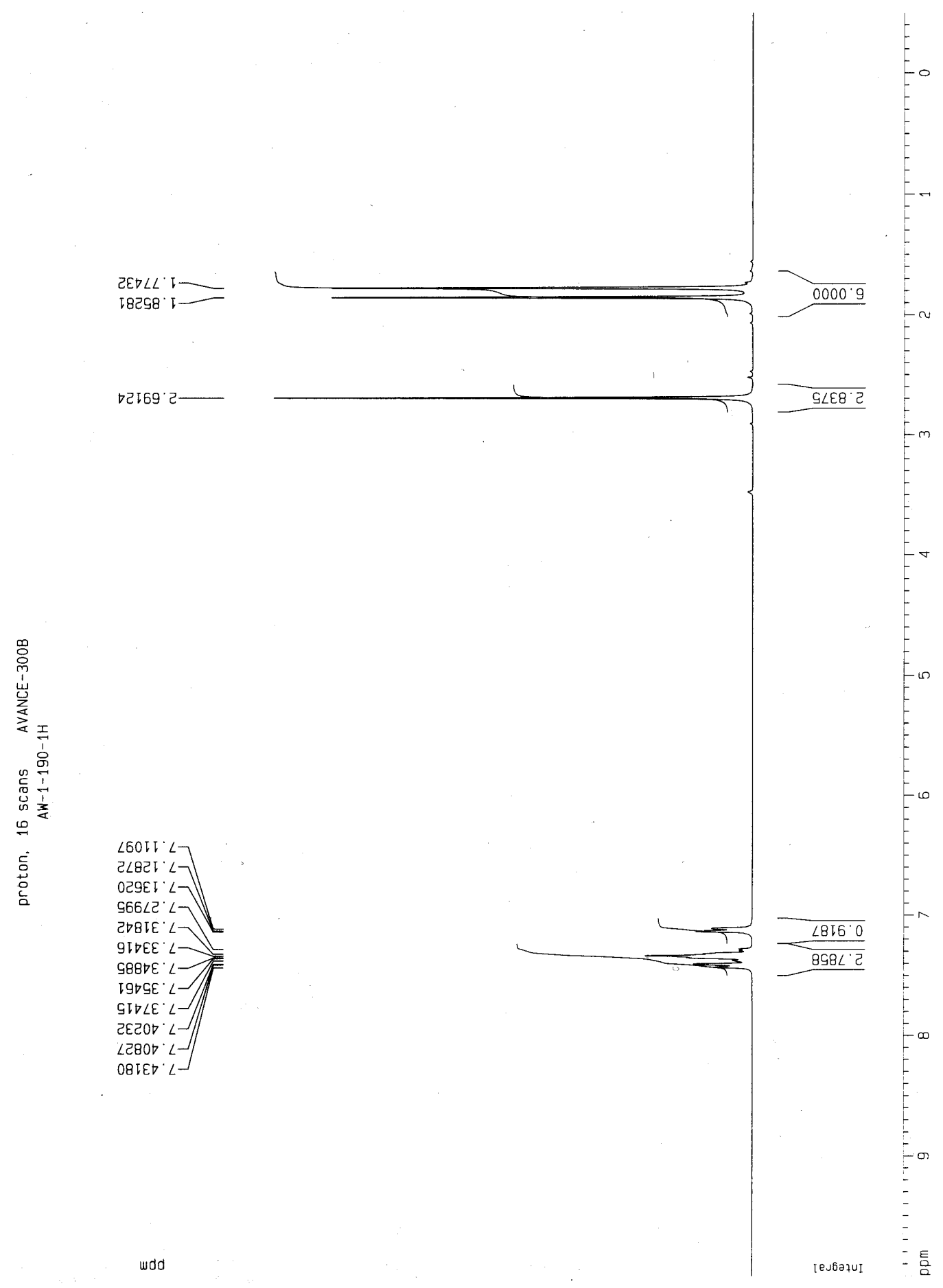




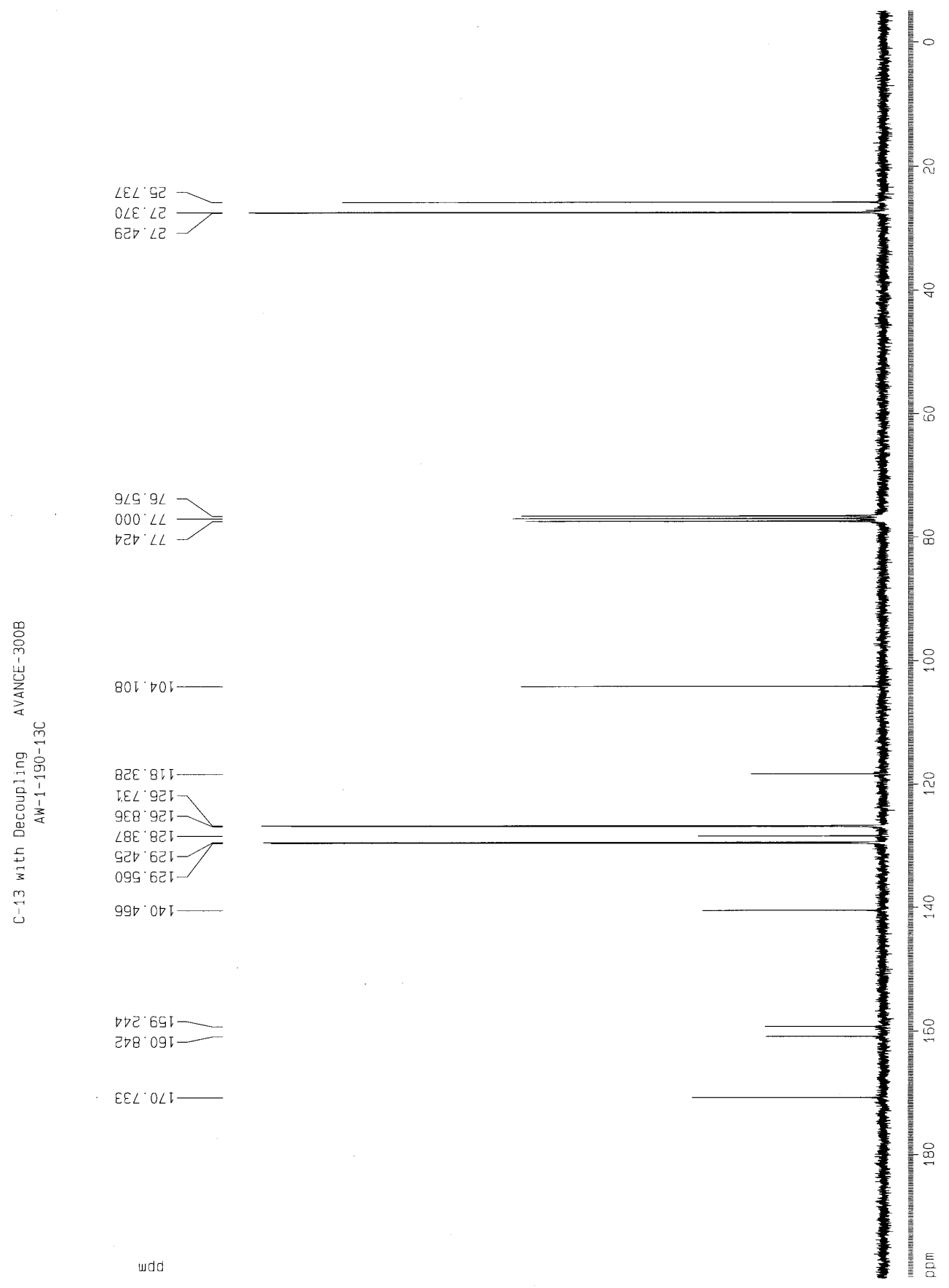




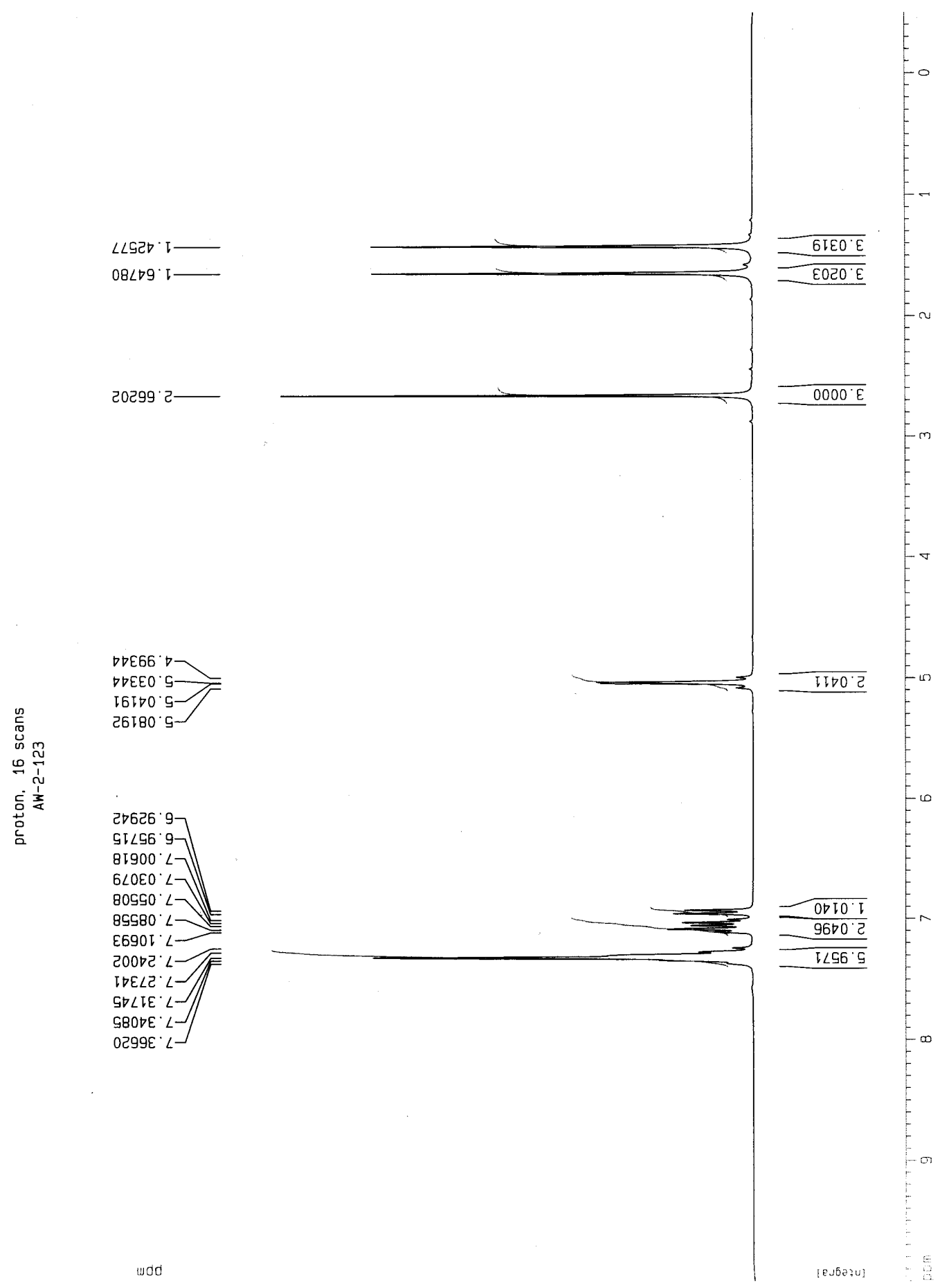



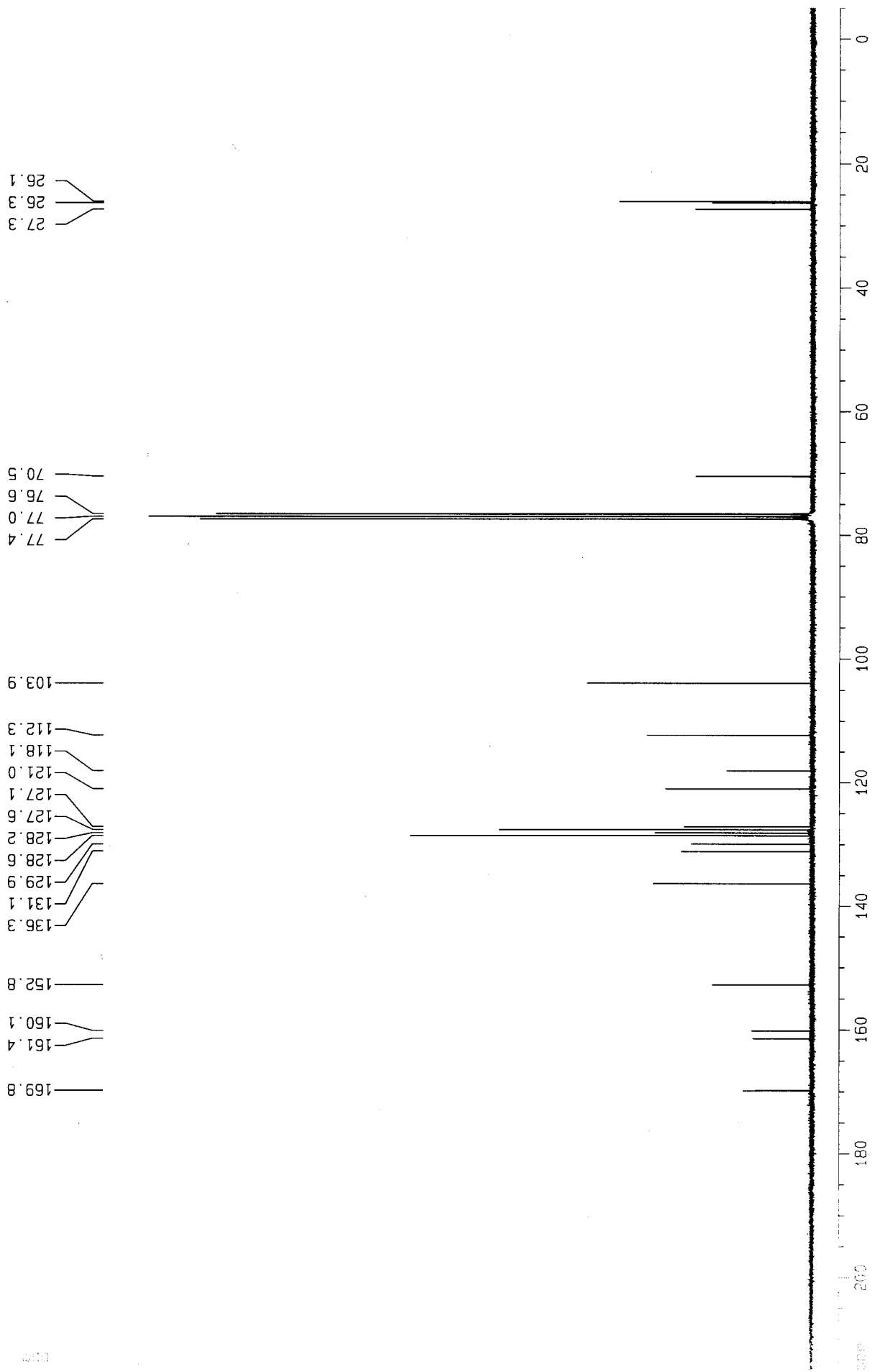


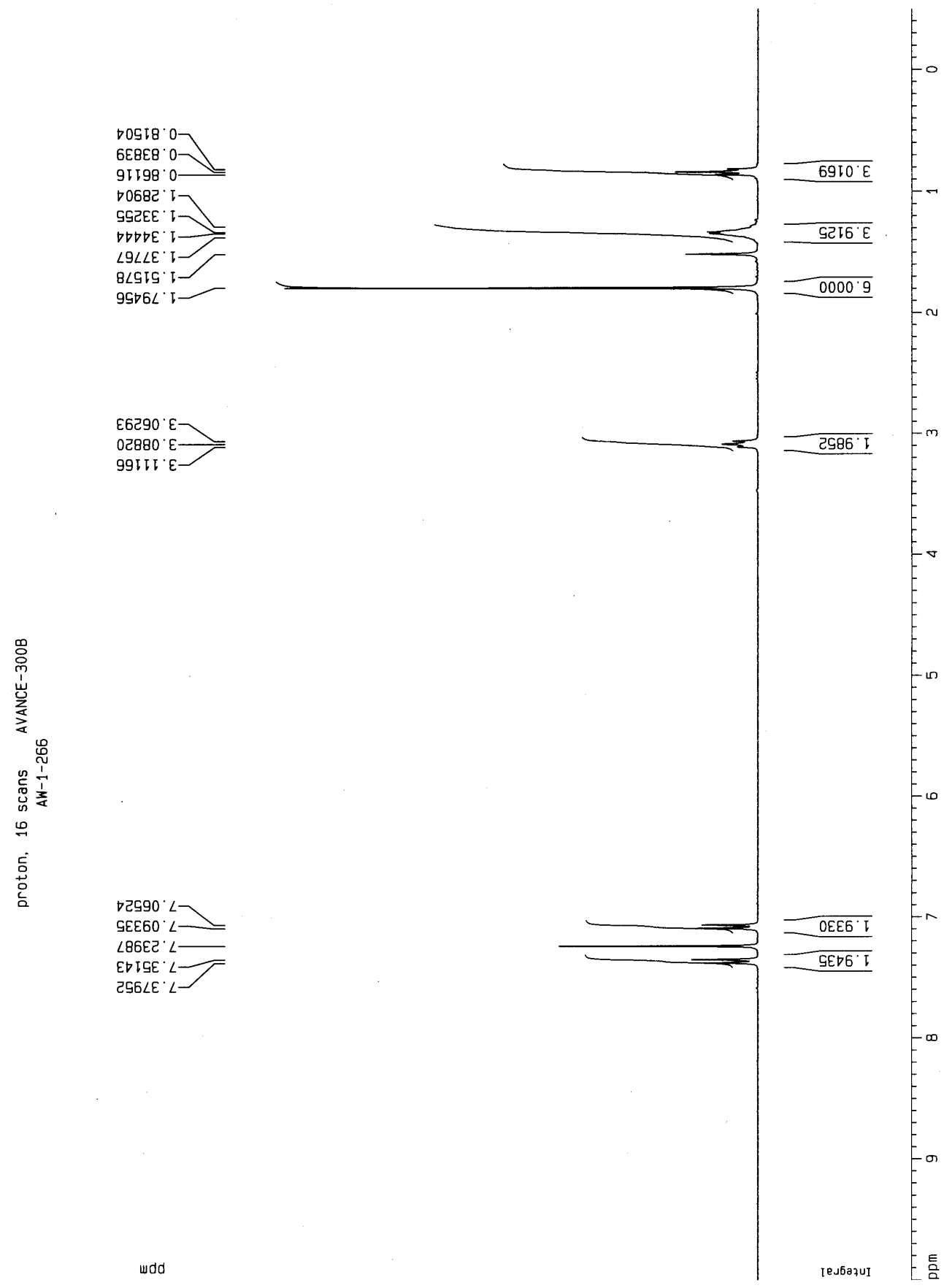




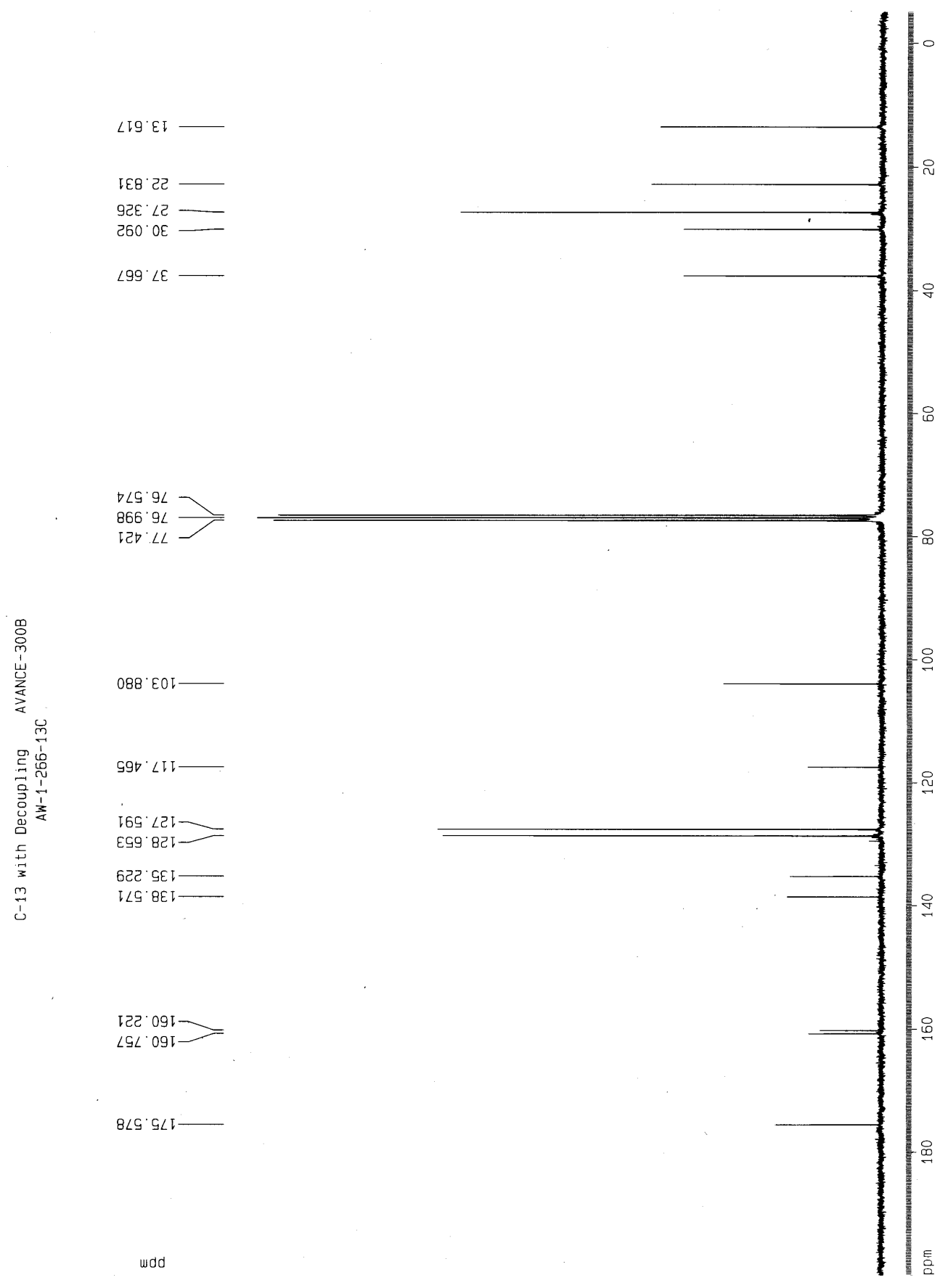




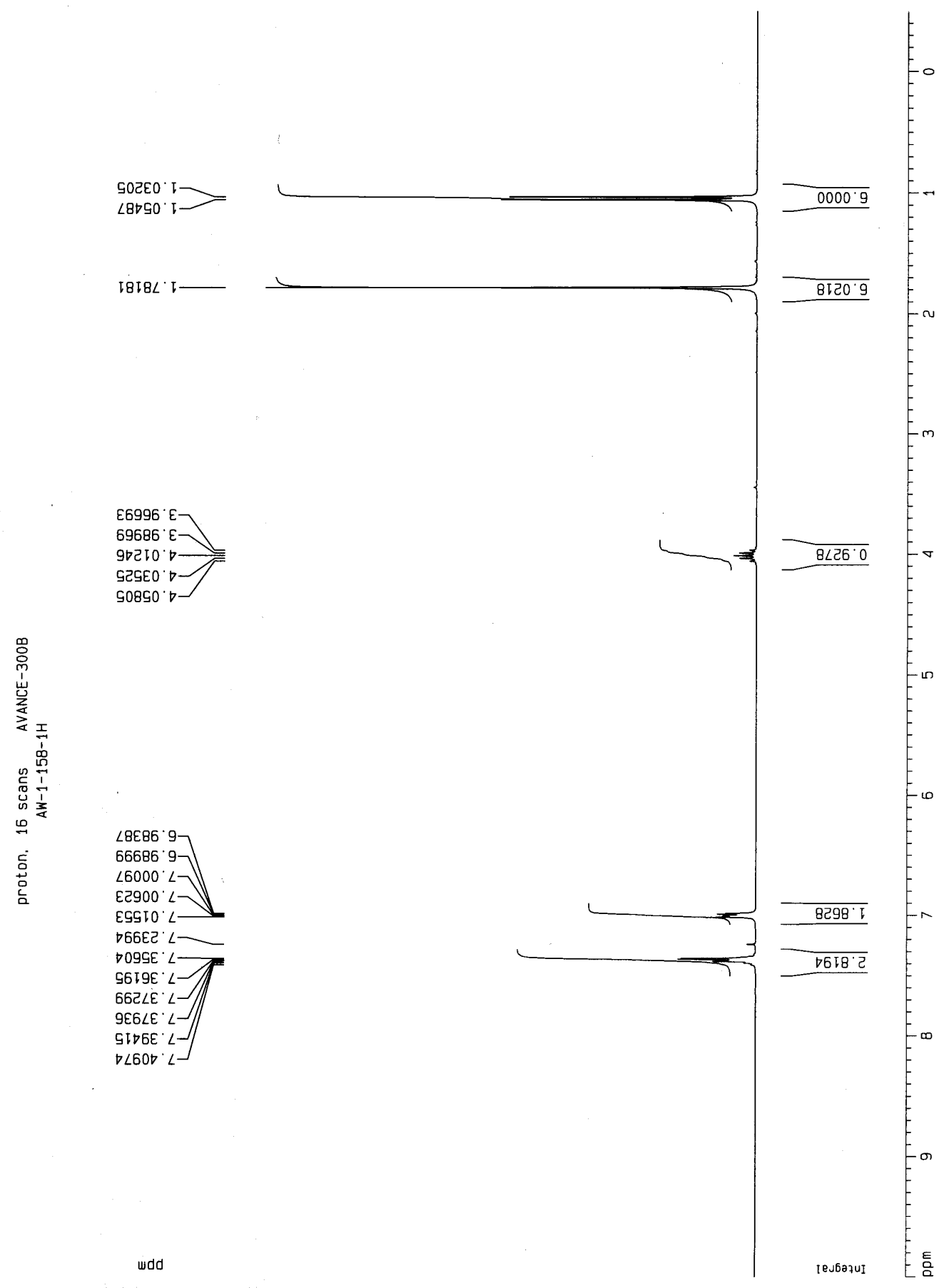




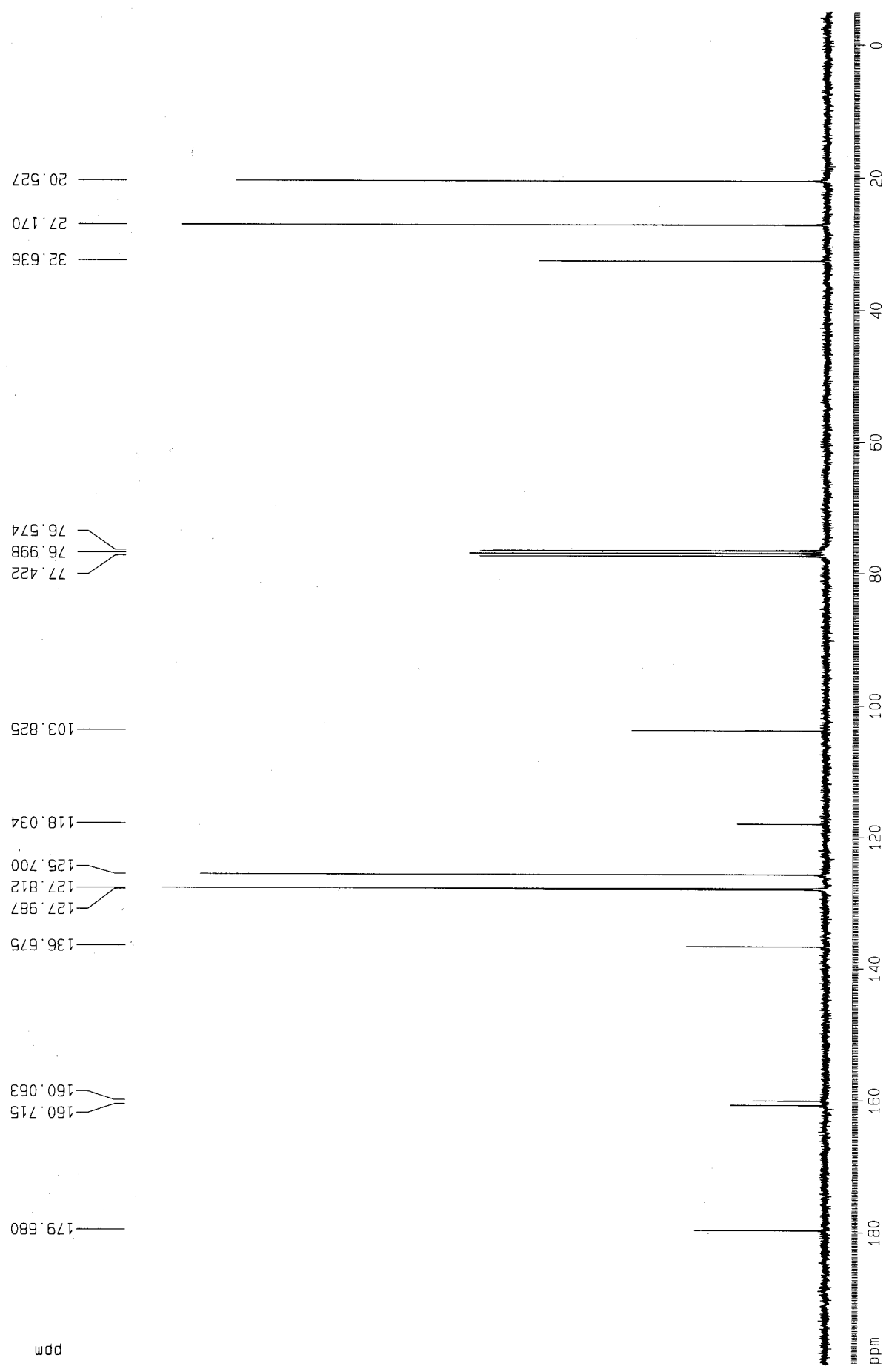




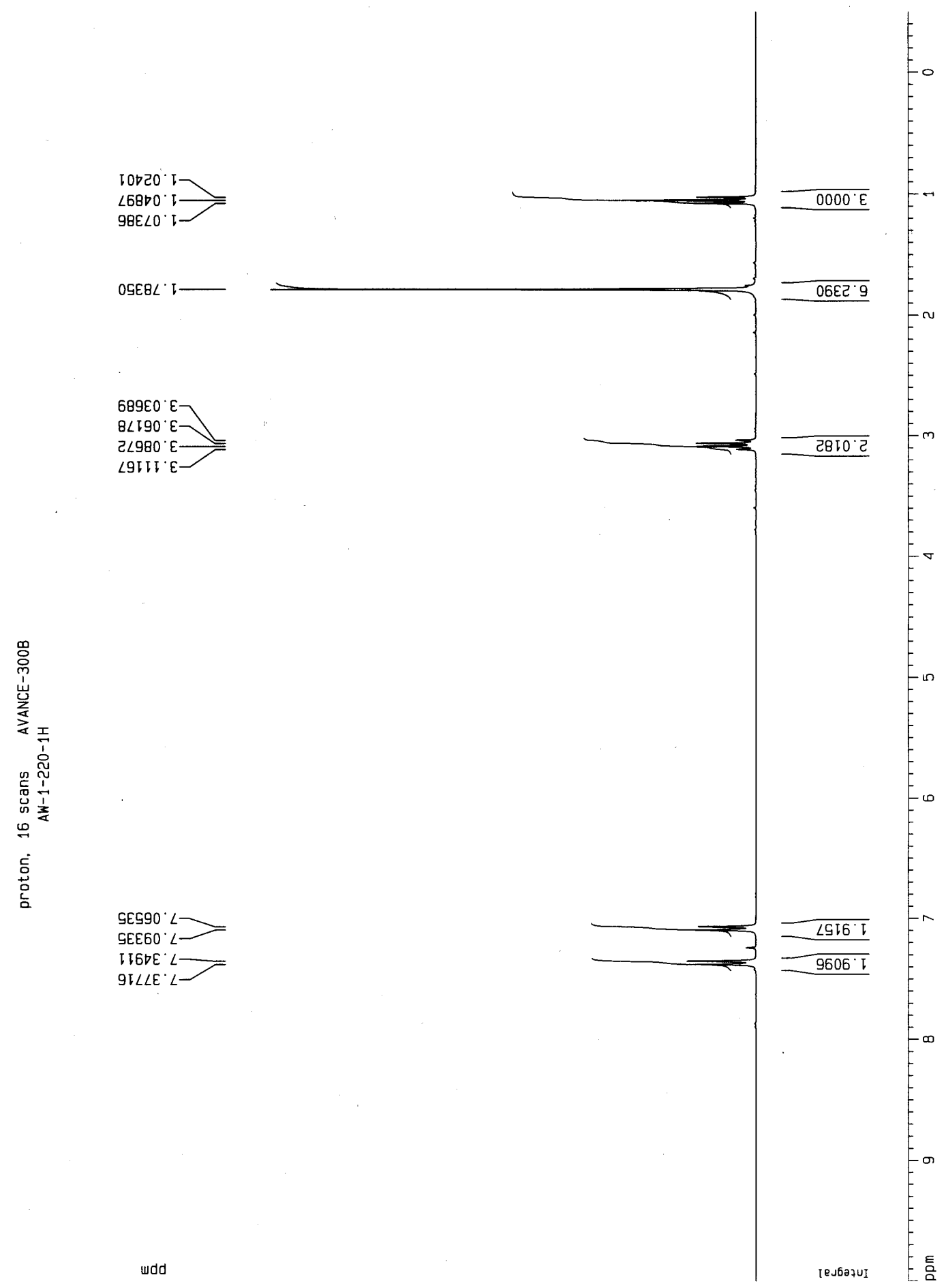




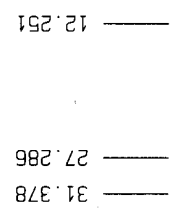

$\varepsilon \angle G^{\circ} 9 \angle$

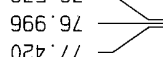

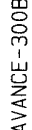

606 EOT -

EGI $\angle I T$

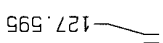

109.8टt

G8T SET-

8๐ट’'BEโ- -

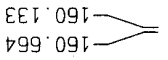

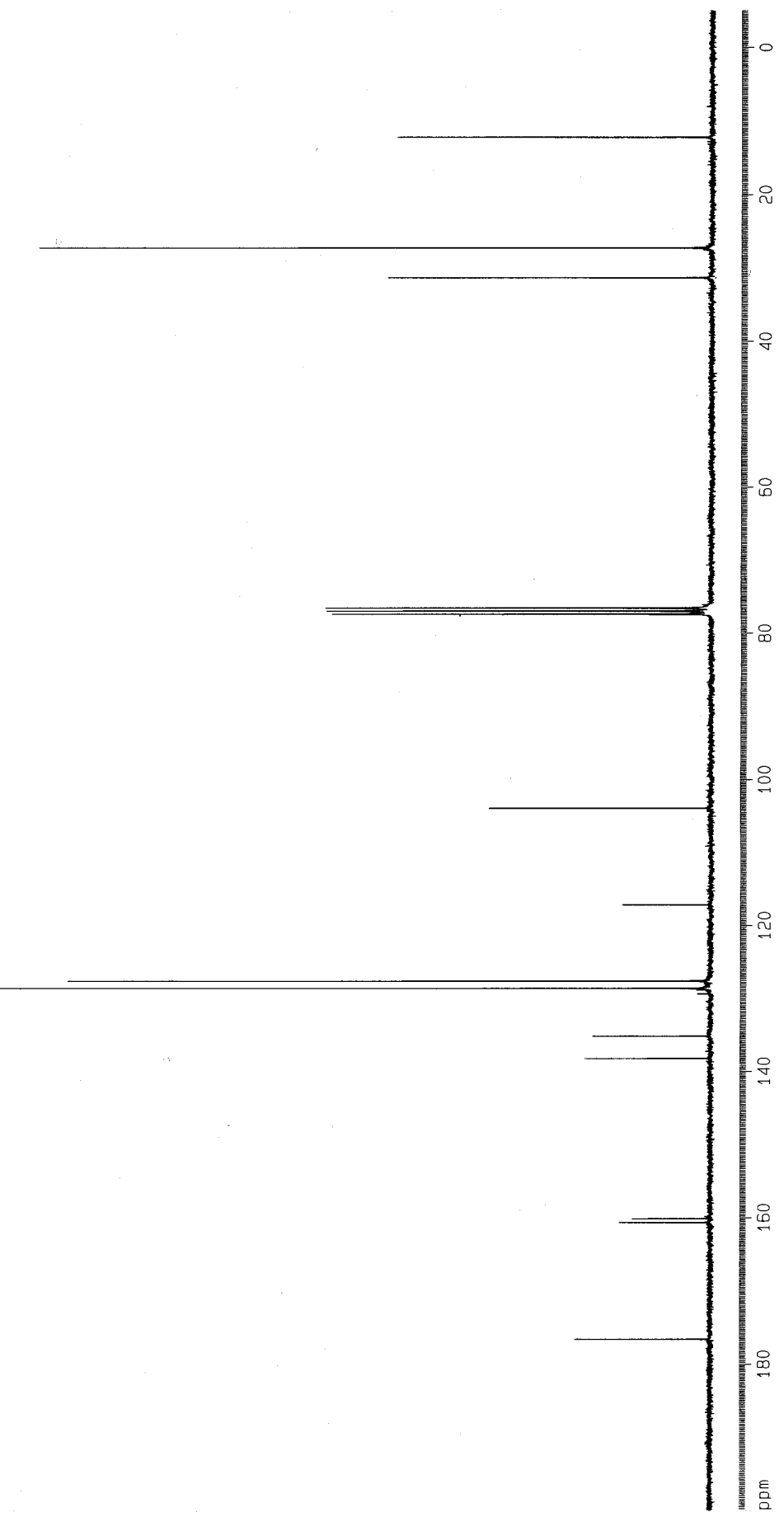

wdd

$89 \cdot 9 \angle 1-$

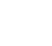




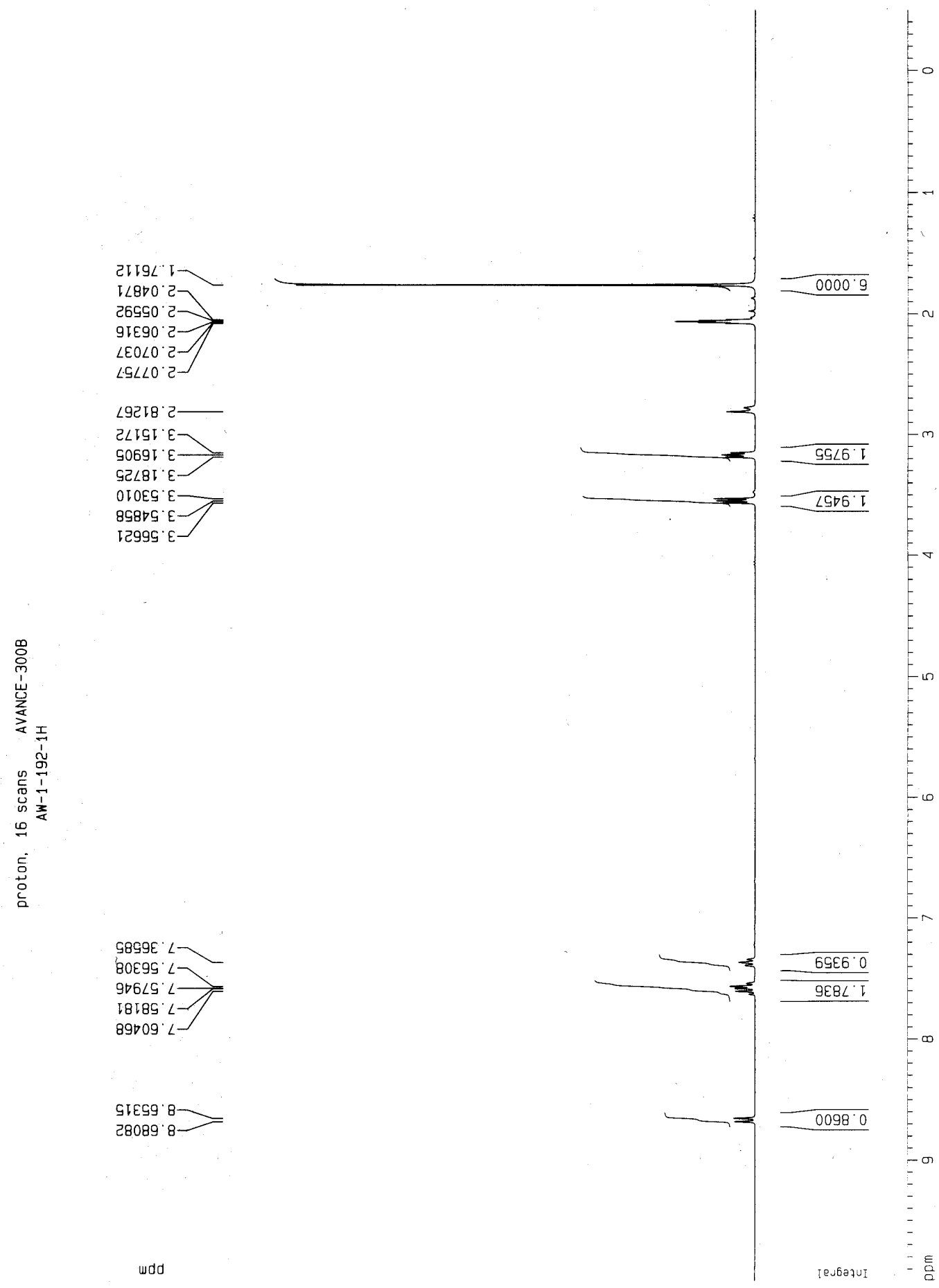




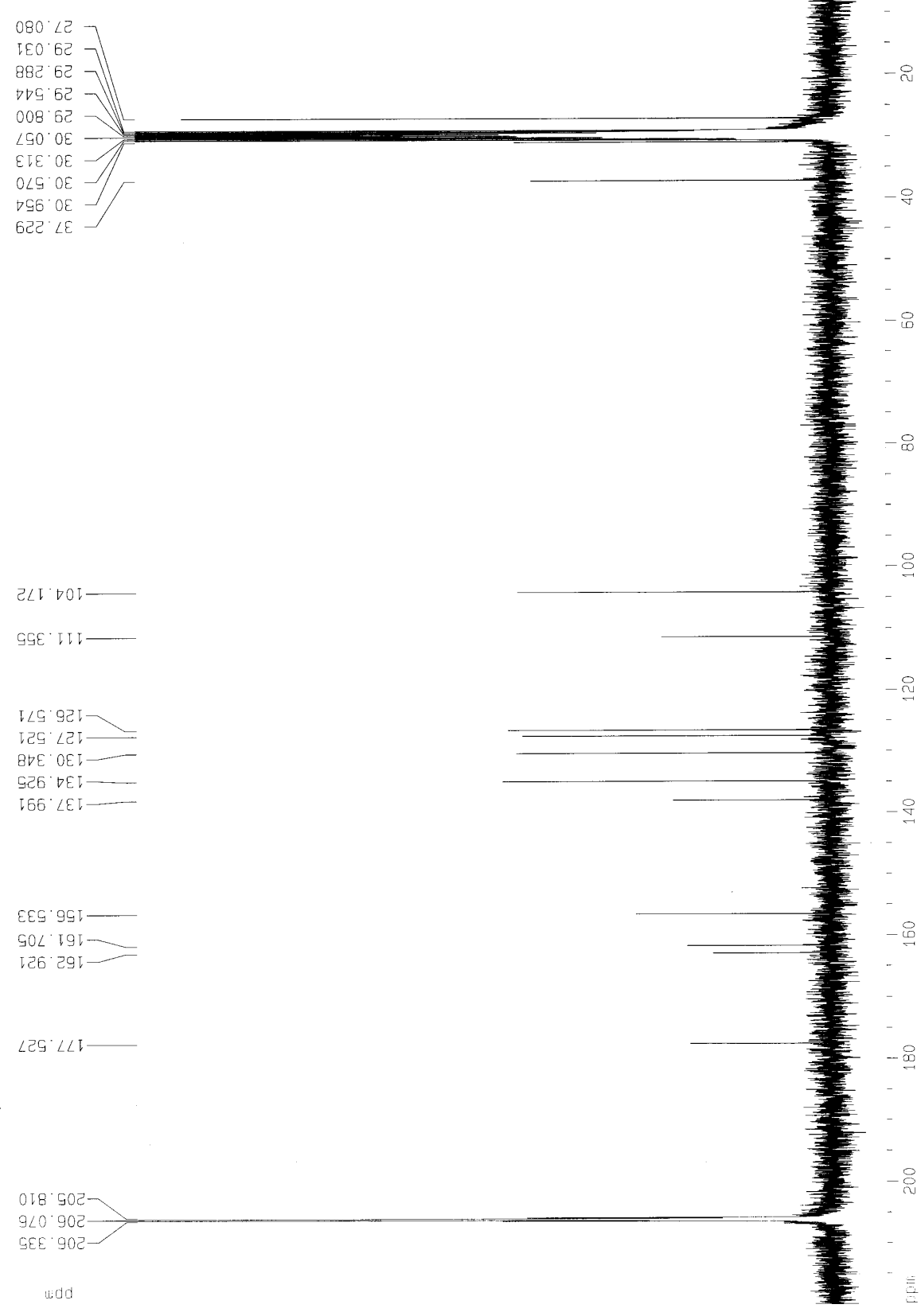



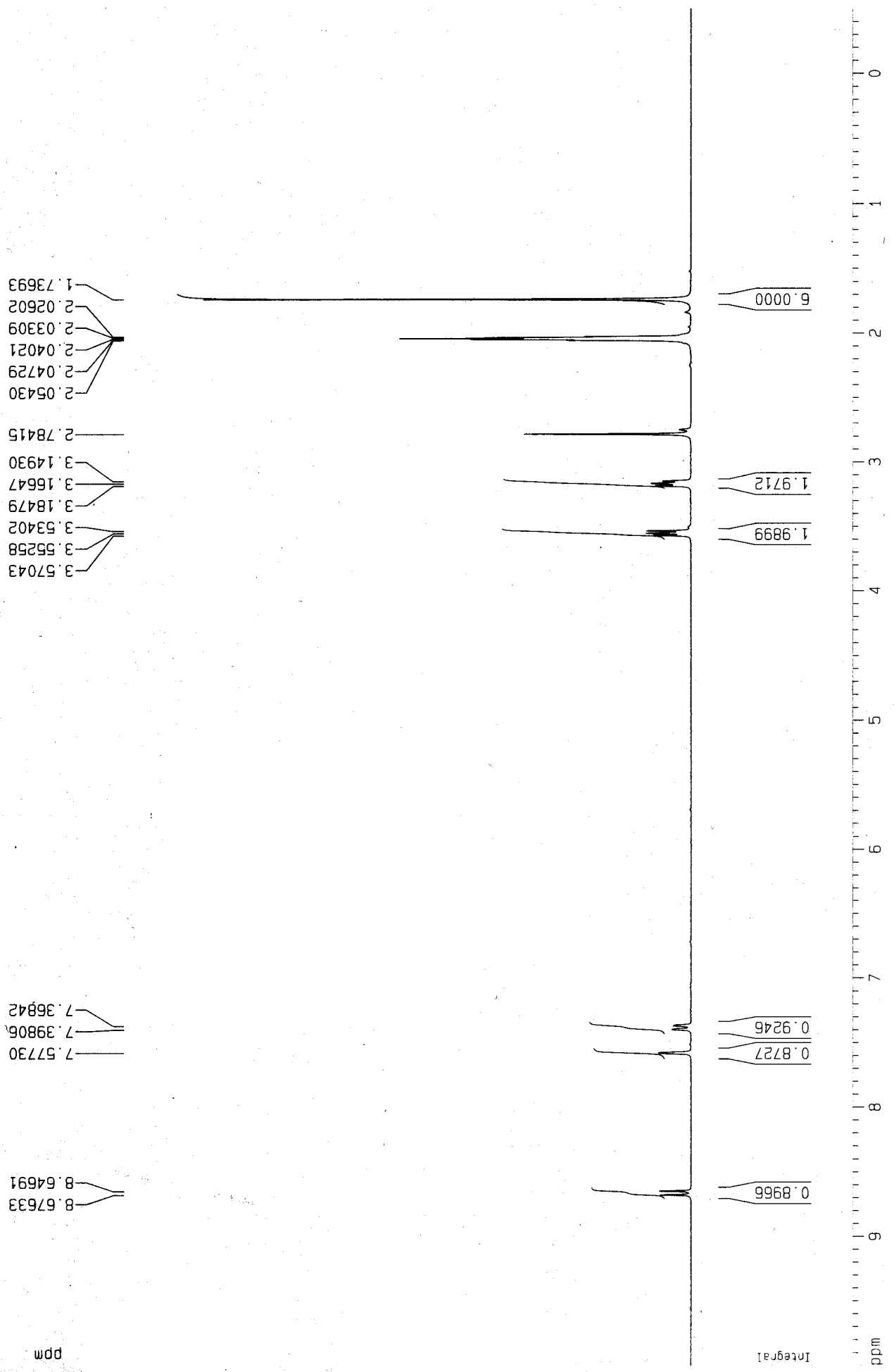

wod

9086E $\angle \longrightarrow$

EE9 $\angle 9.8>=$
(1)

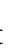




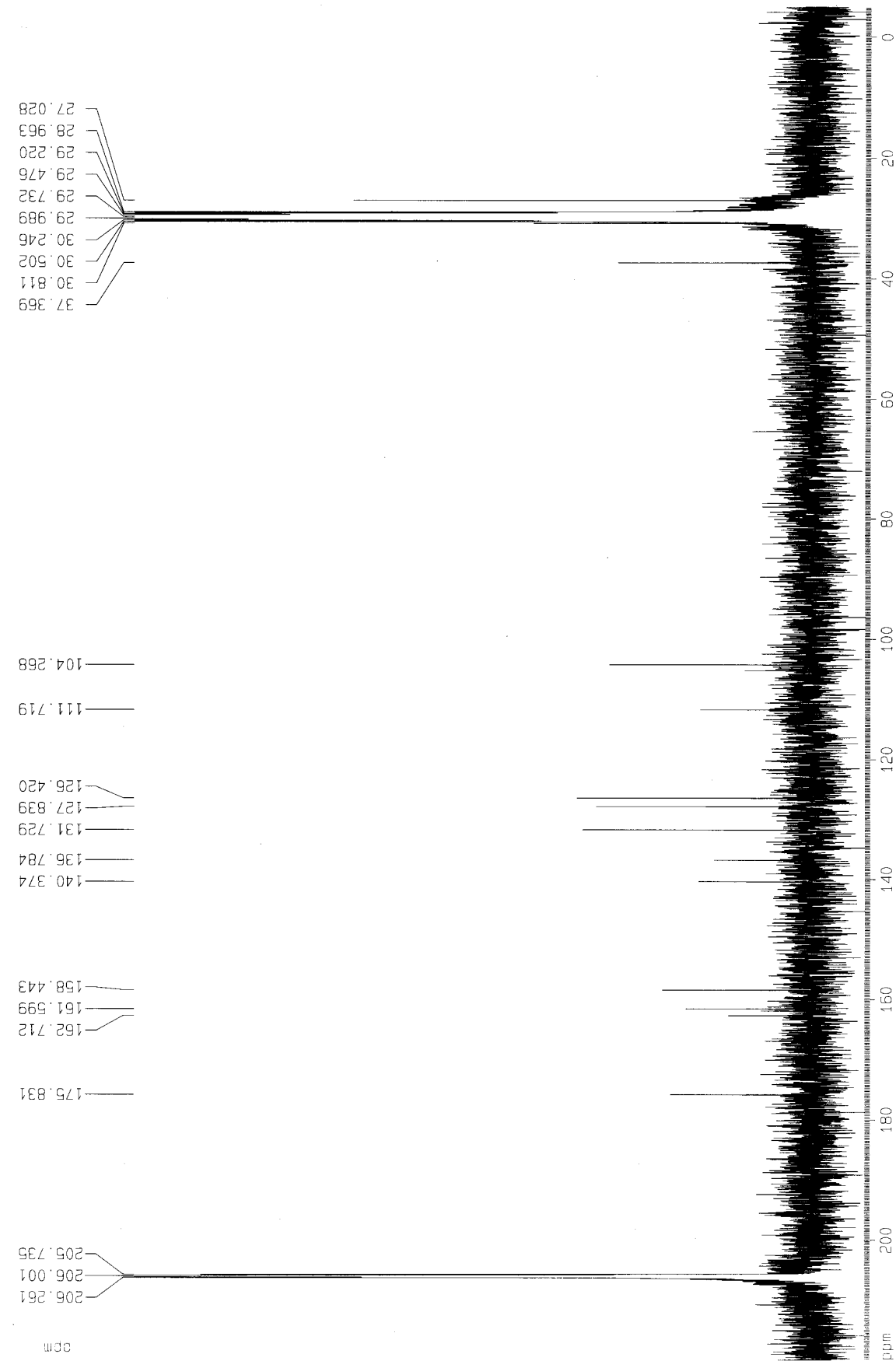

